\title{
Spectra of operators in large $N$ tensor models
}

\author{
Ksenia Bulycheva, ${ }^{1}$ Igor R. Klebanov, ${ }^{1,2}$ Alexey Milekhin, ${ }^{1}$ and Grigory Tarnopolsky ${ }^{1}$ \\ ${ }^{1}$ Department of Physics, Princeton University, Princeton, New Jersey 08544, USA \\ ${ }^{2}$ Princeton Center for Theoretical Science, Princeton University, Princeton, New Jersey 08544, USA
}

(Received 1 September 2017; published 29 January 2018)

\begin{abstract}
We study the operators in the large $N$ tensor models, focusing mostly on the fermionic quantum mechanics with $O(N)^{3}$ symmetry which may be either global or gauged. In the model with global symmetry, we study the spectra of bilinear operators, which are in either the symmetric traceless or the antisymmetric representation of one of the $O(N)$ groups. In the symmetric traceless case, the spectrum of scaling dimensions is the same as in the Sachdev-Ye-Kitaev (SYK) model with real fermions; it includes the $h=2$ zero mode. For the operators antisymmetric in the two indices, the scaling dimensions are the same as in the additional sector found in the complex tensor and SYK models; the lowest $h=0$ eigenvalue corresponds to the conserved $O(N)$ charges. A class of singlet operators may be constructed from contracted combinations of $m$ symmetric traceless or antisymmetric two-particle operators. Their two-point functions receive contributions from $m$ melonic ladders. Such multiple ladders are a new phenomenon in the tensor model, which does not seem to be present in the SYK model. The more typical $2 k$-particle operators do not receive any ladder corrections and have quantized large $N$ scaling dimensions $k / 2$. We construct pictorial representations of various singlet operators with low $k$. For larger $k$, we use available techniques to count the operators and show that their number grows as $2^{k} k$ !. As a consequence, the theory has a Hagedorn phase transition at the temperature which approaches zero in the large $N$ limit. We also study the large $N$ spectrum of low-lying operators in the Gurau-Witten model, which has $O(N)^{6}$ symmetry. We argue that it corresponds to one of the generalized SYK models constructed by Gross and Rosenhaus. Our paper also includes studies of the invariants in large $N$ tensor integrals with various symmetries.
\end{abstract}

DOI: 10.1103/PhysRevD.97.026016

\section{INTRODUCTION AND SUMMARY}

Models where the degrees of freedom are tensors of rank $r>2$ offer the possibility of large $N$ limits dominated by the so-called melon diagrams, if the interactions are chosen appropriately [1-11]. In models where the tensor indices are distinguishable, so that the symmetry group is $O(N)^{r}$ for example, the proofs of melonic limits have been available for several years. ${ }^{1}$ During the recent months, interest in the melonic large $N$ tensor models has been boosted by their connections $[17,18]$ with the SachdevYe-Kitaev model [19-22] and its generalizations [23], as well as by connections with the large $N$ matrix models [24]. In particular, the Schwinger-Dyson (S-D) equations which

\footnotetext{
${ }^{1}$ There is recent evidence $[12,13]$ that the melon dominance extends even to theories with a single $O(N)$ symmetry group, which are similar to the tensor models [14-16] considered in the early 1990s.

Published by the American Physical Society under the terms of the Creative Commons Attribution 4.0 International license. Further distribution of this work must maintain attribution to the author(s) and the published article's title, journal citation, and DOI. Funded by SCOAP.
}

determine the scaling dimensions of a class of bilinear operators $[22,23,25-27]$ have been shown to be identical in the tensor and Sachdev-Ye-Kitaev (SYK) models [18].

In this paper, we continue exploration of the large $N$ tensor models, in particular the $O(N)^{3}$ symmetric model of Ref. [18], which appears to be the minimal quantum mechanical model possessing the melonic limit. ${ }^{2}$ This model has $N^{3}$ anticommuting degrees of freedom, $\psi^{a b c}$, where $a, b, c=1, \ldots, N$. In the model with global symmetry, the operators may be classified according to the group representations. In Sec. III, we study the spectra of two-particle operators, which are either symmetric traceless or antisymmetric under two indices belonging to the same $O(N)$ group. We find that the spectrum of symmetric traceless operators (3.5) is the same as that in the SYK model with real fermions; in particular, it includes the $h=2$ zero mode which plays an important role in the dual gravitational dynamics [28-30]. While in the SYK model there is one $h=2$ zero mode, in the $O(N)^{3}$ tensor model, it appears with multiplicity $1+\frac{3}{2}(N-1)(N+2)$. For the

\footnotetext{
${ }^{2}$ Our work may be generalized to similar models with higher rank tensors, but we will not do this explicitly here.
} 
operators antisymmetric in the two indices (3.6), the spectrum is identical to the additional sector found in the complex tensor and SYK models [18,31-36]; it includes the $h=0$ eigenvalue with multiplicity $\frac{3}{2} N(N-1)$ corresponding to the conserved $O(N)^{3}$ charges.

An attractive feature of the tensor models is that the global symmetry may be gauged $[17,18]$; this restricts the operator spectrum to the invariant ones only. The "Regge trajectory" of two-particle operators $\psi^{a b c} \partial_{t}^{2 n+1} \psi^{a b c}$ is clearly not the full set of $O(N)^{3}$ invariant operators; there are vastly more operators which may be constructed by multiplying an even number of tensors and contracting all the indices [18]. In Sec. IV, we explicitly construct and draw pictorial representations of such operators (these pictures are analogous to the Feynman diagrams in the theory of three scalar fields $\varphi_{i}$ with interaction vertex $\left.\varphi_{1} \varphi_{2} \varphi_{3}\right)$. Using the techniques developed in Refs. [37-40] (see also Ref. [41]), we will calculate the number of $(2 k)$ particle operators and show that it grows asymptotically as $2^{k} k$ !. As a consequence, the theory has a Hagedorn phase transition at the temperature $\sim 1 / \log N$, which we discuss in Sec. IX. Our work is similar in spirit to the classification of invariants in the $d=0$ tensor models [2,42-46], but some of our specific results appear to be new. Working with the quantum mechanical model of real 3-tensors introduces some subtleties and cancellations: for example, in the $O(N)^{3}$ fermionic model all the six-particle operators vanish due to the Fermi statistics, while the number of ten-particle operators is strongly reduced compared to the similar bosonic model. In Sec. VIII, we also count the invariants in $d=0$ bosonic models. In addition to the real tensors with $O(N)^{3}$ symmetry, we study the complex tensor theories with $U(N)^{3}$ and $U(N)^{2} \times O(N)$ symmetries, as well as the symmetric traceless and fully antisymmetric rank-3 tensors under a single $O(N)$ group.

Beyond classifying the invariant operators, it is important to determine their infrared scaling dimensions. We begin work on this in Sec. V and point out that there is a large class of $2 k$-particle operators of which the large $N$ scaling dimensions are simply additive, i.e. $k / 2$. This is because the melonic ladders contribute only to $1 / N$ corrections. However, although less generic, there are operators of which the dimensions are not simply quantized. While the Regge trajectory operators studied in Refs. [18,22,23,25-27] receive single ladder contributions, there are operators of which the two-point functions have multiladder contributions. Since a ladder may contain an $h=2$ zero mode, the $m$-ladder diagram seems to produce a low-temperature enhancement by $(\beta J)^{m}$. This may be an important physical effect in the melonic tensor models, the detailed analysis of which we leave for the future.

Besides our analysis of the spectra of $O(N)^{3}$ symmetric models, we make some comments about the $O(N)^{6}$ symmetric Gurau-Witten model [17]. Some features of its spectrum are identical to those in the $q=4, f=4$
Gross-Rosenhaus flavored generalization [23] of the SYK model. The connections of the Gurau-Witten model with this Gross-Rosenhaus model have been also noted using combinatorial analysis in Ref. [47].

After this paper was completed, we became aware of the interesting Ref. [48], which has some overlap with our results.

\section{COMMENTS ON THE $O(N)^{3}$ SYMMETRIC FERMIONIC TENSOR QUANTUM MECHANICS}

Let us consider the quantum mechanical model of a real anticommuting 3-tensor $\psi^{a b c}$ with the action [18]

$S=\int d t\left(\frac{i}{2} \psi^{a b c} \partial_{t} \psi^{a b c}+\frac{1}{4} g \psi^{a_{1} b_{1} c_{1}} \psi^{a_{1} b_{2} c_{2}} \psi^{a_{2} b_{1} c_{2}} \psi^{a_{2} b_{2} c_{1}}\right)$.

The three indices, each of which runs from 1 to $N$, are treated as distinguishable, and the Majorana fermions satisfy the anticommutation relations

$$
\left\{\psi^{a b c}, \psi^{a^{\prime} b^{\prime} c^{\prime}}\right\}=\delta^{a a^{\prime}} \delta^{b b^{\prime}} \delta^{c c^{\prime}}
$$

This model is a somewhat simplified version of the $O(N)^{6}$ symmetric Gurau-Witten model [17]. Both are in the class of 3-tensor models which possess a "melonic" large $N$ limit where $J=g N^{3 / 2}$ is held fixed [1-11]. The large $N$ model is nearly conformal in the IR [19,22]; for example, the twopoint function is

$$
\begin{aligned}
& \left\langle T\left(\psi^{a b c}\left(t_{1}\right) \psi^{a^{\prime} b^{\prime} c^{\prime}}\left(t_{2}\right)\right)\right\rangle \\
& \quad=-\delta^{a a^{\prime}} \delta^{b b^{\prime}} \delta^{c c^{\prime}}\left(\frac{1}{4 \pi g^{2} N^{3}}\right)^{1 / 4} \frac{\operatorname{sgn}\left(t_{1}-t_{2}\right)}{\left|t_{1}-t_{2}\right|^{1 / 2}} .
\end{aligned}
$$

The model (2.1) has the $O(N)_{1} \times O(N)_{2} \times O(N)_{3}$ symmetry under the replacement ${ }^{3}$

$$
\psi^{a b c} \rightarrow M_{1}^{a a^{\prime}} M_{2}^{b b^{\prime}} M_{3}^{c c^{\prime}} \psi^{a^{\prime} b^{\prime} c^{\prime}}
$$

$M_{1} \in O(N)_{1}, \quad M_{2} \in O(N)_{2}, \quad M_{3} \in O(N)_{3}$.

As far as the group $O(N)_{1}$ is concerned, we may think of $b$ and $c$ as flavor indices; therefore, $\psi^{a b c}$ produces $N^{2}$ flavors of real fermions in the fundamental of $O(N)_{1}$. An analogous picture applies to $O(N)_{2}$ and $O(N)_{3}$. The three sets of $S O(N)$ symmetry charges are

\footnotetext{
${ }^{3}$ More generally, we could consider a model with $O\left(N_{1}\right) \times$ $O\left(N_{2}\right) \times O\left(N_{3}\right)$ symmetry, where $a$ runs from 1 to $N_{1}, b$ runs from 1 to $N_{2}$, and $c$ runs from 1 to $N_{3}$. This may be thought of as a model of a large number $N_{2}$ of $N_{1} \times N_{3}$ matrices [24].
} 


$$
\begin{aligned}
Q_{1}^{a_{1} a_{2}} & =\frac{i}{2}\left[\psi^{a_{1} b c}, \psi^{a_{2} b c}\right], \\
Q_{2}^{b_{1} b_{2}} & =\frac{i}{2}\left[\psi^{a b_{1} c}, \psi^{a b_{2} c}\right], \\
Q_{3}^{c_{1} c_{2}} & =\frac{i}{2}\left[\psi^{a b c_{1}}, \psi^{a b c_{2}}\right] .
\end{aligned}
$$

The gauging of $S O(N)_{1} \times S O(N)_{2} \times S O(N)_{3}$ sets these charges to zero; this restricts the operators to the invariant ones, where all the indices are contracted. In the ungauged model (2.1), a more general class of operators is allowed, and they can be classified according to representations of the $S O(N)_{1} \times S O(N)_{2} \times S O(N)_{3}$.

Each $O(N)$ group includes parity transformations (axis reflections) $P_{a_{0}}$ : for a given $a_{0}, P_{a_{0}}$ sends $\psi^{a_{0} b c} \rightarrow-\psi^{a_{0} b c}$ for all $b, c$ and leaves all $\psi^{a_{1} b c}, a_{1} \neq a_{0}$ invariant. In a physical language, these are "big" gauge transformations, and operators should be invariant under them. Therefore, we can build operators using $\psi^{a b c}$ and the delta symbol $\delta^{a a^{\prime}}$ only. In the case of the $S O(N)$ gauge group, one can use the fully antisymmetric tensor $\epsilon_{a_{1} \ldots a_{N}}$ as well; it is invariant under $S O(N)$ but changes its sign under the parity transformations. Because of this, there are additional "long" operators containing at least $N$ fields, like

$$
O_{\text {long }}=\epsilon_{a_{1} \ldots a_{N}} \epsilon_{b_{1} \ldots b_{N}} \epsilon_{c_{1} \ldots c_{N}} \prod_{j=1}^{N} \psi^{a_{j} b_{j} c_{j}} .
$$

The difference between gauging $O(N)$ and $S O(N)$ becomes negligible in the large $N$ limit.

Let us define three operations which permute pairs of the $O(N)$ symmetry groups (and thus interchange indices in the tensor field), while also reversing the direction of time,

$$
\begin{array}{ll}
s_{a b}: \psi^{a b c} \rightarrow \psi^{b a c}, & t \rightarrow-t ; \\
s_{b c}: \psi^{a b c} \rightarrow \psi^{a c b}, & t \rightarrow-t ; \\
s_{a c}: \psi^{a b c} \rightarrow \psi^{c b a}, & t \rightarrow-t .
\end{array}
$$

Each of these transformations preserves the equations of motion for the $\psi^{a b c}$ field,

$\dot{\psi}^{a b c}=i g\left(\psi^{3}\right)^{a b c}, \quad\left(\psi^{3}\right)^{a b c} \equiv \psi^{a b_{1} c_{1}} \psi^{a_{1} b c_{1}} \psi^{a_{1} b_{1} c}$.

The Hamiltonian, including a quantum shift due to (2.2),

$$
\begin{aligned}
H & =-\frac{1}{4} g \psi^{a_{1} b_{1} c_{1}} \psi^{a_{1} b_{2} c_{2}} \psi^{a_{2} b_{1} c_{2}} \psi^{a_{2} b_{2} c_{1}}+\frac{g N^{4}}{16} \\
& =-\frac{1}{4} g\left[\psi^{a_{1} b_{1} c_{1}}, \psi^{a_{1} b_{2} c_{2}}\right]\left[\psi^{a_{2} b_{1} c_{2}}, \psi^{a_{2} b_{2} c_{1}}\right],
\end{aligned}
$$

changes sign under each of the transformations $s_{a b}, s_{b c}, s_{a c}$ (this is discussed in Sec. IV). This means that these transformations are unitary; they preserve $e^{i H t}$. In contrast, the usual time reversal transformation is antiunitary because it also requires complex conjugation $i \rightarrow-i$.

The $O(N)^{3}$ invariant operators form representations under the permutation group $S_{3}$, which acts on the three $O(N)$ symmetry groups (it contains the elements $s_{a b}, s_{b c}$, and $s_{a c}$ ). For example, $H$ is in the degree 1 "sign representation" of $S_{3}$; it changes sign under any pair interchange but preserves its sign under a cyclic permutation.

It is also interesting to study the spectrum of eigenstates of the Hamiltonian for small values of $N$; first steps on this were made in Refs. [49-51]. When gauging the $O(N)^{3}$ symmetry, one needs to worry about the $Z_{2}$ anomaly, which affects the gauged $O(N)$ quantum mechanics with an odd number of flavors of real fermions in the fundamental representation [52,53]. Since for each of the three $O(N)$ groups we find $N^{2}$ flavors of fundamental fermions, the gauged model is consistent for even $N$ but is anomalous for odd $N .^{4}$ This means that, for odd $N$, the spectrum does not contain states which are invariant under $O(N)^{3}$ (for $N=3$, this can be seen via an explicit diagonalization of the Hamiltonian (2.12) [49]).

\section{COMPOSITE OPERATORS AND SCHWINGER-DYSON EQUATIONS}

The scaling dimensions of a class of bilinear operators may be extracted from the four-point function [18]

$$
\left\langle\psi^{a_{1} b_{1} c_{1}}\left(t_{1}\right) \psi^{a_{1} b_{1} c_{1}}\left(t_{2}\right) \psi^{a_{2} b_{2} c_{2}}\left(t_{3}\right) \psi^{a_{2} b_{2} c_{2}}\left(t_{4}\right)\right\rangle
$$

and factorizing it in the channel where $t_{1} \rightarrow t_{2}$ and $t_{3} \rightarrow t_{4}$. A class of melonic ladder graphs appears in this channel in the large $N$ limit; it may be summed by means of a Schwinger-Dyson equation. The singlet bilinear operators

$$
\mathcal{O}_{n}=\psi^{a b c} \partial_{t}^{2 n+1} \psi^{a b c}, \quad n=0,1,2, \ldots
$$

form a Regge trajectory. Their scaling dimensions are the same as in the SYK model $[19,22]$, and they have been extensively analyzed in the literature [23,25-27]. The dimensions are determined by the equation

$$
g(h)=-\frac{3}{2} \frac{\tan \left(\frac{\pi}{2}\left(h-\frac{1}{2}\right)\right)}{h-1 / 2}=1,
$$

and the first few solutions are $h=2,3.77,5.68, \ldots$ As pointed out in Ref. [18], the model also contains a multitude of multiparticle singlet operators. As we will see, some special combinations of the multiparticle

\footnotetext{
${ }^{4}$ We are grateful to E. Witten for pointing this out to us.
} 


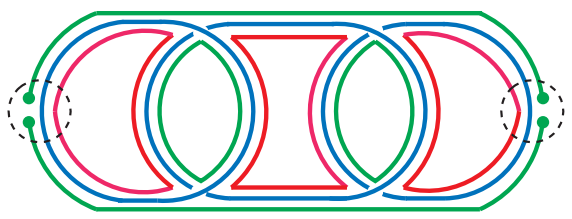

FIG. 1. A ladder contribution to the two-point function of a bilinear operator with two pairs of indices contracted, $\mathcal{O}^{c_{1} c_{2}}$. It is not suppressed in the large $N$ limit.

operators are related by the equations of motion to the operators (3.2), but most multiparticle operators are genuinely new.

Interestingly, there are also certain nonsinglet operators which are renormalized by the melonic ladder diagrams. This can be seen, for example, from the four-point function

$$
\left\langle\psi^{a_{1} b_{1} c_{1}}\left(t_{1}\right) \psi^{a_{2} b_{1} c_{1}}\left(t_{2}\right) \psi^{a_{1} b_{2} c_{2}}\left(t_{3}\right) \psi^{a_{2} b_{2} c_{2}}\left(t_{4}\right)\right\rangle
$$

factorized in the channel $t_{1} \rightarrow t_{2}$ and $t_{3} \rightarrow t_{4}$. As shown in Fig. 1, all the melonic ladders again make nonvanishing contributions in the large $N$ limit. Here, we find two classes of nonsinglet bilinear operators: those symmetric and traceless in $a_{1}$ and $a_{2}$ and those antisymmetric. The $\frac{1}{2}(N-1)(N+2)$ symmetric traceless operators under $O(N)_{1}$,

$$
\begin{aligned}
\mathcal{O}_{n}^{\left(a_{1} a_{2}\right)}= & \psi^{a_{1} b c} \partial_{t}^{2 n+1} \psi^{a_{2} b c}+\psi^{a_{2} b c} \partial_{t}^{2 n+1} \psi^{a_{1} b c} \\
& -\frac{2}{N} \delta^{a_{1} a_{2}} \psi^{a b c} \partial_{t}^{2 n+1} \psi^{a b c}
\end{aligned}
$$

where $n=0,1,2, \ldots$, have the same spectrum as the singlet bilinears (3.2), which is determined by (3.3). Of course, there are analogous operators $\mathcal{O}_{n}^{\left(b_{1} b_{2}\right)}$ and $\mathcal{O}_{n}^{\left(c_{1} c_{2}\right)}$ that are symmetric traceless under $O(N)_{2}$ and $O(N)_{3}$, respectively. Thus, the symmetric traceless operators present in the ungauged model contain the $h=2$ zero mode with multiplicity $\frac{3}{2}(N-1)(N+2)$; this appears to imply a significant physical difference between the ungauged $O(N)^{3}$ model and the SYK model. ${ }^{5}$ While in the gauged model such bilinear operators are projected out, we may form singlet combinations out of their products; such operators have an interesting feature that they are renormalized by multiple ladders. For example, in Sec. V. we will encounter operators related by the equation of motion to $\mathcal{O}_{0}^{\left(a_{1} a_{2}\right)} \mathcal{O}_{0}^{\left(a_{1} a_{2}\right)}$, so they are renormalized by double ladders. The pictorial representations of these operators may be found in column 2 of Fig. 9.

There are also the $\frac{1}{2} N(N-1)$ operators in the antisymmetric two-index representation of $O(N)_{1}$,

\footnotetext{
${ }^{5}$ We are grateful to Shiraz Minwalla for very useful discussions on this; see Ref. [48].
}

$$
\mathcal{O}_{n}^{\left[a_{1} a_{2}\right]}=\psi^{a_{1} b c} \partial_{t}^{2 n} \psi^{a_{2} b c}-\psi^{a_{2} b c} \partial_{t}^{2 n} \psi^{a_{1} b c},
$$

and the analogous antisymmetric operators under $O(N)_{2}$ and $O(N)_{3}$. The Schwinger-Dyson equations for these operators are identical to the "symmetric sector" of the complex tensor model [18,31-36]. Their scaling dimensions are determined by

$$
\tilde{g}(h)=-\frac{1}{2} \frac{\tan \left(\frac{\pi}{2}\left(h+\frac{1}{2}\right)\right)}{h-1 / 2}=1 .
$$

The first few solutions of this equation are $h=0,2.65$, $4.58, \ldots$, and each one appears with multiplicity $\frac{3}{2} N(N-1)$. The spectrum includes the special $h=0$ mode corresponding here to the $n=0$ operators, which are the $O(N)^{3}$ charges (2.6).

The four-point function (3.4) may also be factorized in the channel $t_{1} \rightarrow t_{3}$ and $t_{2} \rightarrow t_{4}$. This leads to the spectrum of operators

$$
\mathcal{O}_{m}^{b_{1} c_{1} b_{2} c_{2}}=\psi^{a b_{1} c_{1}} \partial_{t}^{m} \psi^{a b_{2} c_{2}} .
$$

We can see from Fig. 2 that the ladder contribution to this operator is subleading in $1 / N$; the rightmost diagram is of ladder type and is $\sim g^{2} N^{3}$, which is suppressed by a power of $N$ relative to the other two diagrams. Therefore, the large $N$ scaling dimensions of these operators are $1 / 2+m$.

We will adopt a pictorial representation of the operators where the $\psi^{a b c}$ fields are shown as the vertices. The $a$ indices which transform under $O(N)_{1}$ are shown by red lines, the $b$-indices which transform under $\mathrm{O}(\mathrm{N})_{2}$ are shown by blue lines, and the $c$-indices which transform under $\mathrm{O}(\mathrm{N})_{3}$ are shown by green lines. For example, the three charges (2.6) are shown in Fig. 3.

\section{CONSTRUCTION OF $O(N)^{3}$ INVARIANT OPERATORS}

In this section, we study the spectrum of $O(N)^{3}$ invariant operators. Since a time derivative may be removed using the equations of motion (2.11), we may write the operators in a form in which no derivatives are present. The bilinear singlet operator, $\psi^{a b c} \psi^{a b c}$, vanishes classically by the Fermi statistics, while at the quantum level taking into account (2.2), it is a C-number. The first nontrivial operators appear at the quartic level and are shown in Fig. 4 (from here on, we will not be careful about the quantum corrections to operators).

On the left is the "tetrahedron operator" $O_{\text {tetra }}$, which is proportional to the Hamiltonian (2.12):

$$
O_{\text {tetra }}=\psi^{a_{1} b_{1} c_{1}} \psi^{a_{1} b_{2} c_{2}} \psi^{a_{2} b_{1} c_{2}} \psi^{a_{2} b_{2} c_{1}} .
$$

One can check that 

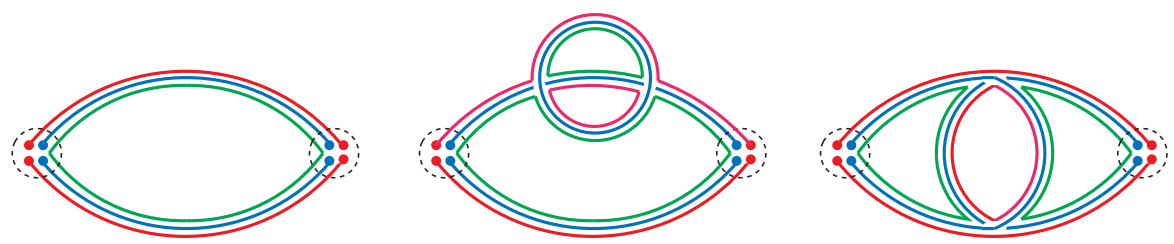

FIG. 2. Different contributions to the two-point function of a bilinear operator with one pair of indices contracted, $\mathcal{O}_{m}^{b_{1} c_{1} b_{2} c_{2}}$. The ladder diagrams, such as the rightmost figure, are suppressed in the large $N$ limit.
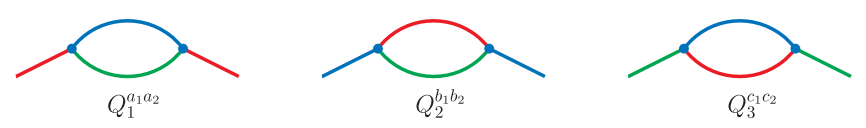

FIG. 3. The $O(N)_{1}, O(N)_{2}$, and $O(N)_{3}$ charges.
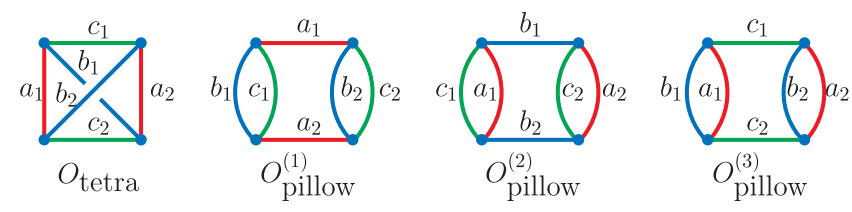

FIG. 4. All the four-particle operators, the tetrahedron and the three pillows, with the index contractions shown explicitly.

$$
\begin{aligned}
s_{b c} O_{\text {tetra }} & =\psi^{a_{1} c_{1} b_{1}} \psi^{a_{1} c_{2} b_{2}} \psi^{a_{2} c_{2} b_{1}} \psi^{a_{2} c_{1} b_{2}} \\
& =\psi^{a_{1} b_{1} c_{1}} \psi^{a_{1} b_{2} c_{2}} \psi^{a_{2} b_{2} c_{1}} \psi^{a_{2} b_{1} c_{2}}=-O_{\text {tetra }}
\end{aligned}
$$

and also that $s_{a b} O_{\text {tetra }}=-O_{\text {tetra }}$ and $s_{a c} O_{\text {tetra }}=-O_{\text {tetra }}$. Thus, the tetrahedron operator $O_{\text {tetra }}$ is in the degree 1 sign representation of $S_{3}$; it changes sign under any pair interchange but preserves its sign under a cyclic permutation.

The three additional operators in Fig. 4, which we denote as $O_{\text {pillow }}^{(1)}, O_{\text {pillow }}^{(2)}$, and $O_{\text {pillow }}^{(3)}$, are the "pillow" operators in the terminology of Refs. $[6,10]$; they contain double lines between a pair of vertices. For example, for $O_{\text {pillow }}^{(1)}$, we have

$O_{\text {pillow }}^{(1)}=-\psi^{a_{1} b_{1} c_{1}} \psi^{a_{2} b_{1} c_{1}} \psi^{a_{1} b_{2} c_{2}} \psi^{a_{2} b_{2} c_{2}}=Q_{1}^{a_{1} a_{2}} Q_{1}^{a_{1} a_{2}}$.

Under the $S_{3}$, the three pillow operators decompose into the trivial representation of degree 1 and the standard representation of degree 2. Since the charges (2.6) commute with the Hamiltonian (2.12), so does each of the three pillow operators. This means that the scaling dimensions of the pillow operators are unaffected by the interactions; i.e., they vanish. In fact, the three pillow operators are simply the quadratic Casimir operators of the three $O(N)$ groups. $^{6}$ The gauging of $O(N)^{3}$ symmetry

\footnotetext{
${ }^{6}$ We thank Dan Roberts and Douglas Stanford for discussions on this.
}

sets the charges (2.6) to zero, so the pillow operators do not appear in the gauged model.

Using the equations of motion (2.11) we see that the operator $O_{\text {tetra }}$ is related by the equation of motion to the operator $\psi^{a b c} \partial_{t} \psi^{a b c}$,

$$
O_{\text {tetra }}=\psi^{a b c}\left(\psi^{3}\right)^{a b c} \propto \psi^{a b c} \partial_{t} \psi^{a b c} .
$$

If we iterate the use of the equation of motion (2.11), then all derivatives in an operator may be traded for extra $\psi$ fields. Thus, a complete basis of operators may be constructed by multiplying some number $2 k$ of $\psi$ fields and contracting all indices. In this approach, there is a unique operator with $k=2(n+1)$ which is equal to the Regge trajectory operator $\psi^{a b c} \partial_{t}^{2 n+1} \psi^{a b c}$. For $n=0$, this operator is $O_{\text {tetra }}$, which is proportional to the Hamiltonian; for $n=1$, it will be constructed explicitly in Sec. IVA.

All the six-particle operators are represented in Fig. 5, but due to the Fermi statistics, all of them vanish. Even if this were not the case, the operators in the first three columns would vanish in the gauged model because they contain insertions of the charges (2.6). Let us demonstrate the vanishing of the two operators in the last column in detail. The first operator

$O_{6}^{(1)}=\psi^{a_{1} b_{1} c_{1}} \psi^{a_{1} b_{2} c_{2}} \psi^{a_{2} b_{1} c_{2}} \psi^{a_{2} b_{3} c_{3}} \psi^{a_{3} b_{3} c_{1}} \psi^{a_{3} b_{2} c_{3}}$

may be written as
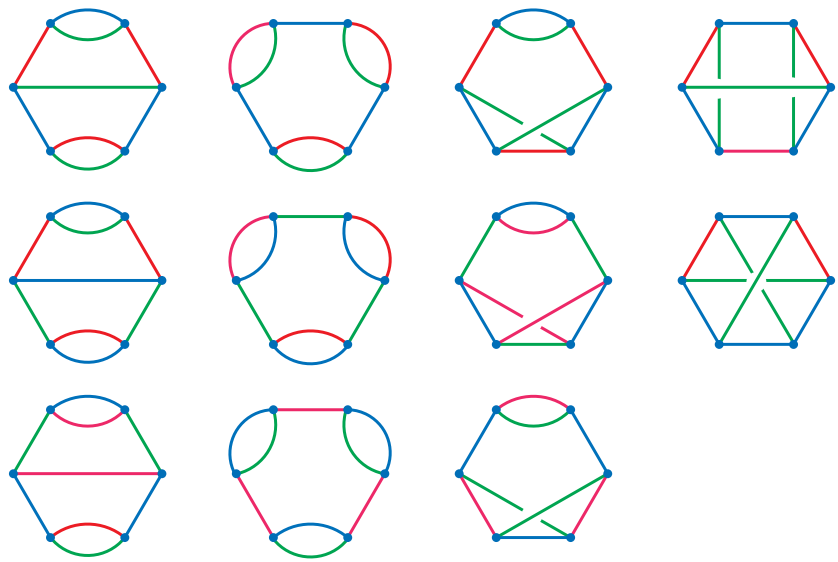

FIG. 5. All six-particle operators. They are present in the scalar model but vanish in the fermionic model. 

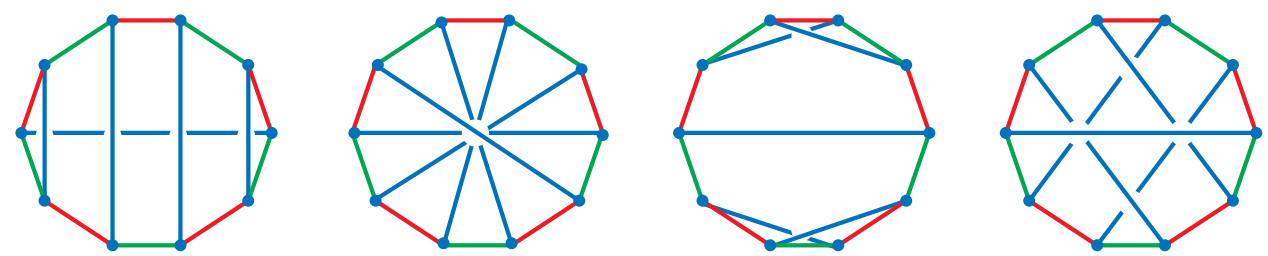

FIG. 6. Some ten-particle operators which vanish in the fermionic model.

$$
O_{6}^{(1)}=\left(\psi^{3}\right)^{a_{2} b_{2} c_{1}}\left(\psi^{3}\right)^{a_{2} b_{2} c_{1}}=0 .
$$

This may be seen by cutting the diagram for this operator in Fig. 5 along the vertical symmetry axis. To show that

$O_{6}^{(2)}=\psi^{a_{1} b_{1} c_{1}} \psi^{a_{1} b_{2} c_{2}} \psi^{a_{2} b_{2} c_{3}} \psi^{a_{2} b_{3} c_{1}} \psi^{a_{3} b_{3} c_{2}} \psi^{a_{3} b_{1} c_{3}}$

also vanishes, we may permute the first two $\psi$ fields to write it as

$O_{6}^{(2)}=-\psi^{a_{1} b_{2} c_{2}} \psi^{a_{1} b_{1} c_{1}} \psi^{a_{2} b_{2} c_{3}} \psi^{a_{2} b_{3} c_{1}} \psi^{a_{3} b_{3} c_{2}} \psi^{a_{3} b_{1} c_{3}}$.

After relabeling $b_{1} \leftrightarrow b_{2}, \quad c_{1} \leftrightarrow c_{2}$, and $a_{2} \leftrightarrow a_{3}$, we observe that the rhs equals $-O_{6}^{(2)}$. Therefore, $O_{6}^{(2)}=-O_{6}^{(2)}=0$.

One may wonder if the vanishing extends to the tenparticle operators. We have checked that the operators shown in Fig. 6 all vanish; this is due to the reflection symmetry present for these operators. For example, the left operator in Fig. 6 vanishes because it may be written as $\left(\psi^{5}\right)^{a b c}\left(\psi^{5}\right)^{a b c}$, which may be seen by cutting the diagram along the vertical symmetry axis. We note that

$$
\left(\psi^{5}\right)^{a b c}=g^{-2} \partial_{t}^{2} \psi^{a b c} .
$$

Similarly, by cutting the third diagram in Fig. 6 along its vertical symmetry axis, we see that the corresponding
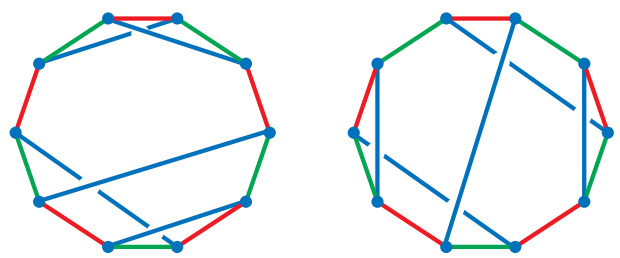

FIG. 7. Some nonvanishing ten-particle operators. operator may be written as $\left(\psi^{5}\right)^{a b_{1} b_{2} b_{3} b_{4} c}\left(\psi^{5}\right)^{a b_{1} b_{2} b_{3} b_{4} c}$ which obviously vanishes as well. This argument extends to all the reflection symmetric $(4 n+2)$-particle diagrams.

However, not all ten-particle operators vanish. For example, the operators shown in Fig. 7 do not have a reflection symmetry, and we have checked that they do not vanish.

Let us note that each gauge invariant operator, where all the indices are contracted, corresponds to a vacuum Feynman diagram in the theory with three scalar fields and interaction $\lambda \varphi_{1} \varphi_{2} \varphi_{3}$ (the three different propagators correspond to the lines of three different colors in our figures). In the theory of bosonic tensors $\phi^{a b c}$, the number of operators made out of $2 k$ fields is precisely the number of distinct Feynman diagrams appearing at order $\lambda^{2 k}$, which grows as $k ! 2^{k}$. In the fermionic model, some of the operators vanish by the Fermi statistics, while others vanish due to the gauge constraint. Nevertheless, we will find that the factorial growth holds also in the fermionic model.

\section{A. Eight-particle operators}

In this section, we explicitly construct all the eightparticle operators without bubble (double line) insertions and exhibit their pictorial representations. Having two vertices connected by a double line corresponds to the insertion of an $O(N)$ charge which vanishes in the gauged model. For this reason, we will omit such operators and list only those where there are no double lines. The possible topologically inequivalent eightparticle operators are shown in Fig. 8; from these, we can obtain other admissible operators by interchanging the colors. In this way, we find 17 inequivalent operators shown in Fig. 9.

Among the eight-particle operators, there are three which may be obtained from the tetrahedral vertex
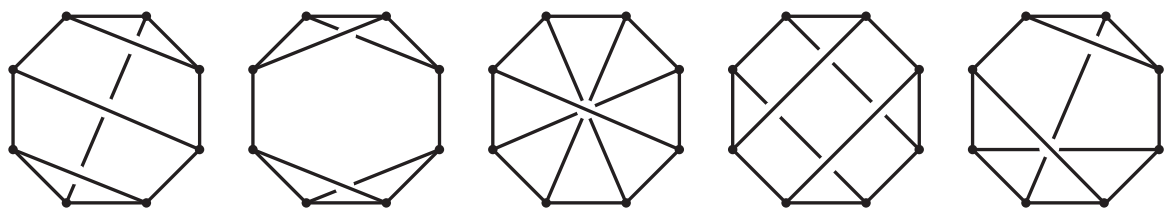

FIG. 8. Eight-particle operator topologies. 

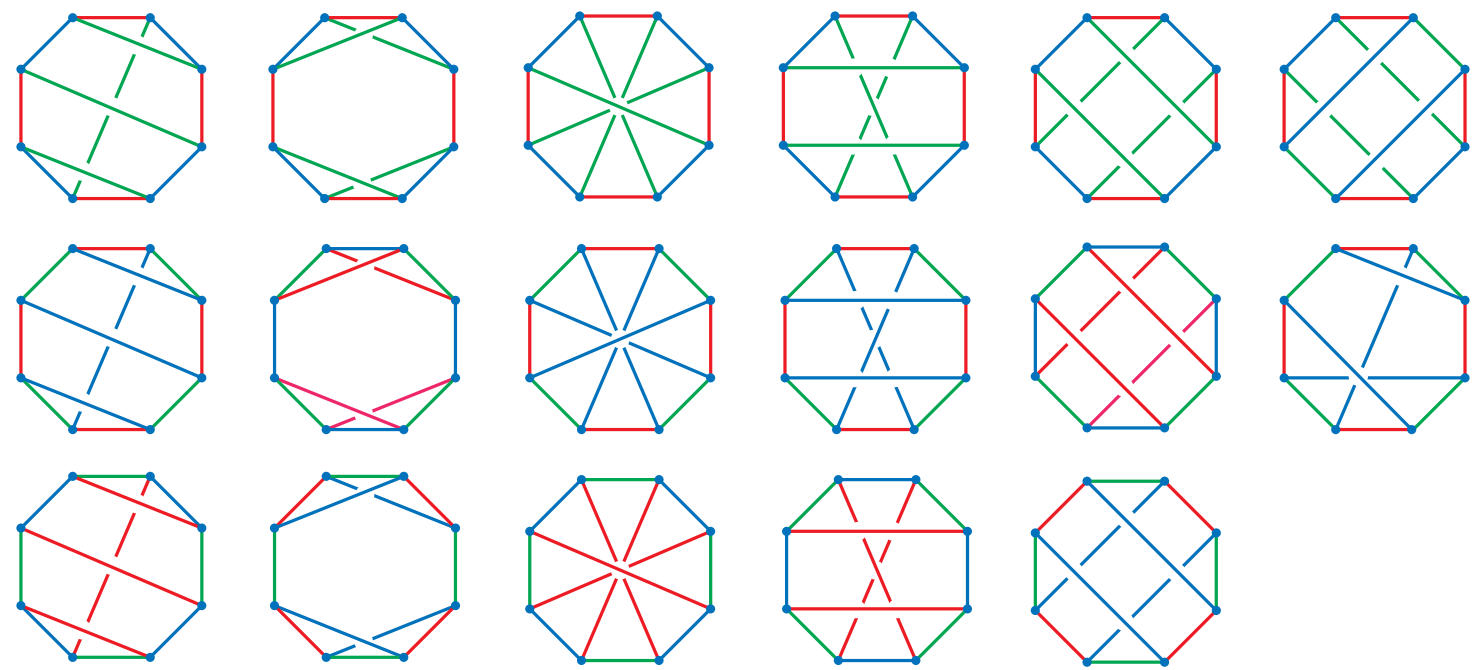

FIG. 9. All eight-particle operators in the fermionic model.

$$
\begin{aligned}
O_{1}= & \psi^{a_{1} b_{1} c_{1}} \psi^{a_{1} b_{2} c_{2}} \psi^{a_{2} b_{2} c_{1}} \psi^{a_{2} b_{4} c_{4}} \psi^{a_{3} b_{3} c_{2}} \\
& \times \psi^{a_{3} b_{1} c_{3}} \psi^{a_{4} b_{4} c_{3}} \psi^{a_{4} b_{3} c_{4}}, \\
O_{2}= & \psi^{a_{1} b_{1} c_{1}} \psi^{a_{1} b_{2} c_{2}} \psi^{a_{2} b_{2} c_{1}} \psi^{a_{2} b_{3} c_{3}} \psi^{a_{3} b_{3} c_{2}} \psi^{a_{3} b_{4} c_{4}} \\
& \times \psi^{a_{4} b_{4} c_{3}} \psi^{a_{4} b_{1} c_{4}}, \\
O_{3}= & \psi^{a_{1} b_{1} c_{1}} \psi^{a_{1} b_{2} c_{2}} \psi^{a_{2} b_{2} c_{1}} \psi^{a_{2} b_{3} c_{3}} \psi^{a_{3} b_{1} c_{3}} \psi^{a_{3} b_{4} c_{4}} \\
& \times \psi^{a_{4} b_{3} c_{4}} \psi^{a_{4} b_{4} c_{2}} .
\end{aligned}
$$

Their pictorial representations are shown in the first column of Fig. 9. Using the equations of motion, we may write them as

$$
\begin{aligned}
& O_{1}=\dot{\psi}^{a_{1} b_{1} c_{1}} \dot{\psi}^{a_{1} b_{2} c_{2}} \psi^{a_{2} b_{1} c_{2}} \psi^{a_{2} b_{2} c_{1}}, \\
& O_{2}=\dot{\psi}^{a_{1} b_{1} c_{1}} \psi^{a_{1} b_{2} c_{2}} \dot{\psi}^{a_{2} b_{1} c_{2}} \psi^{a_{2} b_{2} c_{1}}, \\
& O_{3}=\dot{\psi}^{a_{1} b_{1} c_{1}} \psi^{a_{1} b_{2} c_{2}} \psi^{a_{2} b_{1} c_{2}} \dot{\psi}^{a_{2} b_{2} c_{1}} .
\end{aligned}
$$

It follows that

$$
O_{1}+O_{2}+O_{3} \sim \partial_{t} \psi^{a b c} \partial_{t}^{2} \psi^{a b c},
$$

which up to a total derivative equals the Regge trajectory operator $\psi^{a b c} \partial_{t}^{3} \psi^{a b c}$.

The transformation properties of operators $O_{1}, O_{2}$, and $\mathrm{O}_{3}$ under $\mathrm{S}_{3}$ are

$$
\begin{array}{lll}
s_{b c} O_{3}=-O_{2}, & s_{b c} O_{2}=-O_{3}, & s_{b c} O_{1}=-O_{1}, \\
s_{a c} O_{3}=-O_{1}, & s_{a c} O_{2}=-O_{2}, & s_{a c} O_{1}=-O_{3}, \\
s_{a b} O_{3}=-O_{3}, & s_{a b} O_{2}=-O_{1}, & s_{a b} O_{1}=-O_{2} .
\end{array}
$$

It follows that

$$
\left(s_{a b}, s_{a c}, s_{b c}\right):\left(O_{1}+O_{2}+O_{3}\right) \rightarrow-\left(O_{1}+O_{2}+O_{3}\right) .
$$

Therefore, the operator $\psi^{a b c} \partial_{t}^{3} \psi^{a b c} \sim O_{1}+O_{2}+O_{3}$ is in the degree 1 sign representation of $S_{3}$. The other two linear combinations of operators (4.10), $\mathrm{O}_{1}-\mathrm{O}_{2}$ and $\mathrm{O}_{2}-\mathrm{O}_{3}$, form the standard degree 2 representation of $S_{3}$.

Similarly, we may write down the three operators which correspond to the second column in Fig. 9 (the first of these operators, $\tilde{O}_{1}$, was written down in Ref. [18]):

$$
\begin{aligned}
\tilde{O}_{1}= & \psi^{a_{1} b_{1} c_{1}} \psi^{a_{1} b_{2} c_{2}} \psi^{a_{2} b_{3} c_{3}} \psi^{a_{2} b_{4} c_{4}} \psi^{a_{3} b_{1} c_{3}} \psi^{a_{3} b_{3} c_{1}} \\
& \times \psi^{a_{4} b_{2} c_{4}} \psi^{a_{4} b_{4} c_{2}}, \\
\tilde{O}_{2}= & \psi^{a_{1} b_{1} c_{1}} \psi^{a_{2} b_{1} c_{2}} \psi^{a_{3} b_{2} c_{3}} \psi^{a_{4} b_{2} c_{4}} \psi^{a_{1} b_{3} c_{3}} \psi^{a_{3} b_{3} c_{1}} \\
& \times \psi^{a_{2} b_{4} c_{4}} \psi^{a_{4} b_{4} c_{2}}, \\
\tilde{O}_{3}= & \psi^{a_{1} b_{1} c_{1}} \psi^{a_{2} b_{2} c_{1}} \psi^{a_{3} b_{3} c_{2}} \psi^{a_{4} b_{4} c_{2}} \psi^{a_{3} b_{1} c_{3}} \psi^{a_{1} b_{3} c_{3}} \\
& \times \psi^{a_{4} b_{2} c_{4}} \psi^{a_{2} b_{4} c_{4}} .
\end{aligned}
$$

Via the equations of motion, these operators are related to the bilinear operators defined in (3.5):

$$
\begin{array}{ll}
\tilde{O}_{1} \sim \mathcal{O}_{0}^{\left(a_{1} a_{2}\right)} \mathcal{O}_{0}^{\left(a_{1} a_{2}\right)}, & \tilde{O}_{2} \sim \mathcal{O}_{0}^{\left(b_{1} b_{2}\right)} \mathcal{O}_{0}^{\left(b_{1} b_{2}\right)}, \\
\tilde{O}_{3} \sim \mathcal{O}_{0}^{\left(c_{1} c_{2}\right)} \mathcal{O}_{0}^{\left(c_{1} c_{2}\right)} . &
\end{array}
$$

These relations will be used in the next section.

The action of the discrete symmetries on the operators is

$s_{b c} \tilde{O}_{3}=\tilde{O}_{2}, \quad s_{b c} \tilde{O}_{2}=\tilde{O}_{3}, \quad s_{b c} \tilde{O}_{1}=\tilde{O}_{1}$,

$s_{a c} \tilde{O}_{3}=\tilde{O}_{1}, \quad s_{a c} \tilde{O}_{2}=\tilde{O}_{2}, \quad s_{a c} \tilde{O}_{1}=\tilde{O}_{3}$,

$s_{a b} \tilde{O}_{3}=\tilde{O}_{3}, \quad s_{a b} \tilde{O}_{2}=\tilde{O}_{1}, \quad s_{a b} \tilde{O}_{1}=\tilde{O}_{2}$, 

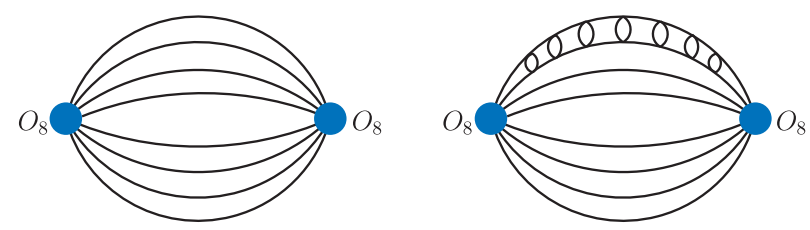

FIG. 10. Diagrammatics for the "typical" operators of which the IR dimensions are quantized. Each line denotes a dressed propagator. a) The melonic diagrams that contribute to the operator two-point functions in the large $N$ limit. b) The ladder diagrams which do not contribute in the large $N$ limit.

so that

$\left(s_{a b}, s_{b c}, s_{a c}\right): \tilde{O}_{1}+\tilde{O}_{2}+\tilde{O}_{3} \rightarrow \tilde{O}_{1}+\tilde{O}_{2}+\tilde{O}_{3}$.

Therefore, this operator is in the trivial representation of $S_{3}$. The other two linear combinations of operators (4.14), $\tilde{O}_{1}-\tilde{O}_{2}$ and $\tilde{O}_{2}-\tilde{O}_{3}$, form the standard degree 2 representation of $S_{3}$. The operators corresponding to the other topologies in Fig. 8 may be written down analogously.

\section{SCALING DIMENSIONS OF MULTIPARTICLE OPERATORS}

We have seen that the tensor models admit a variety of singlet operators. In this section, we discuss their scaling dimensions. Since operators $\mathcal{O}_{m}^{b_{1} c_{1} b_{2} c_{2}}$ defined in (3.8) do not receive ladder contributions in the large $N$ limit, we expect a large class of $m$-particle operators to have the quantized dimensions ${ }^{7}$ :

$$
\Delta_{m}=\frac{m}{4}+\mathcal{O}(1 / N)
$$

This is the dimension of an operator which is not renormalized by ladder diagrams because every pair of tensors has at most one index in common. This situation is illustrated in Fig. 10; the dominant contribution comes from the two operators contracted using the IR two-point function (2.3), and the ladder insertions are suppressed by $1 / N$. We find that this applies to most of the 17 eightparticle operators shown in Fig. 9. The exceptions are operators $O_{i}$ and $\tilde{O}_{i}$, defined in (4.10) and (4.14) and shown in columns 1 and 2. For example, each of the operators $\tilde{O}_{i}$ in column 2 is renormalized by two ladders, as we discuss below.

Thus, the $m / 4$ rule does not apply to all operators; it is violated for the operators of which the two-point functions receive the melonic ladder contributions in the large $N$ limit. One class of such singlet operators is the Regge trajectory we have discussed before:

\footnotetext{
${ }^{7}$ We are very grateful to E. Witten for pointing this out to us.
}

$$
\psi^{a b c} \partial_{t}^{2 n+1} \psi^{a b c}
$$

After applying the equation of motion (2.11), which schematically may be represented as

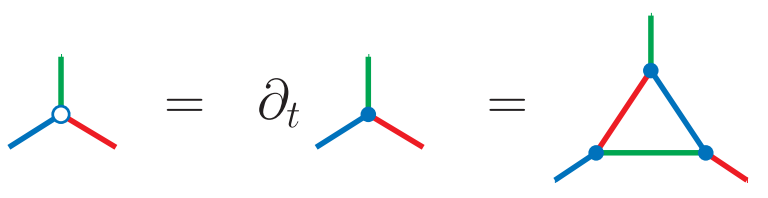

we may represent the Regge trajectory operators in terms of multiparticle operators without derivatives. For example, the $n=0$ operator is equivalent to the four-particle tetrahedron operator $O_{\text {tetra, }}$, while the $n=1$ operator is equivalent to $\mathrm{O}_{1}+\mathrm{O}_{2}+\mathrm{O}_{3}$, as shown in (4.12). The dimensions of such operators come from solving (3.3), so the operator $O_{1}+O_{2}+O_{3}$ has $h \approx 3.77$.

Furthermore, using the equation of motion (5.3), we can relate many additional singlet operators to operators containing derivatives. Let us denote a vertex with $\partial_{t} \psi$ by a white circle. By the equations of motion, we can relate the operators of which the diagram contains triangles with low-order operators containing derivatives. For example, some of the operators which can be written as lower-order operators with derivatives are shown in Fig. 11.

As discussed in Sec. III, some of these operators are renormalized by multiple ladder diagrams. For example, the three four-particle pillow operators, shown in Fig. 4, have dimension $h=0$ because they are squares of the symmetry charges. Similarly, operators $\mathcal{O}_{0}^{\left(a_{1} a_{2}\right)} \mathcal{O}_{0}^{\left(a_{1} a_{2}\right)}$ related by the equation of motion to column 2 of Fig. 9 are renormalized by double ladders as shown in Fig. 12 . One can also see that the correlation function of this operator with four fermionic fields receives a contribution from two ladders as shown in Fig. 13 and 14.

More generally, we may use operators $\mathcal{O}_{n}^{\left(a_{1} a_{2}\right)}$ defined in (3.5) to write down the singlet operators

$$
\mathcal{O}_{n_{1} n_{2}}=\mathcal{O}_{n_{1}}^{\left(a_{1} a_{2}\right)} \mathcal{O}_{n_{2}}^{\left(a_{1} a_{2}\right)}
$$

renormalized by double ladders,

$$
\mathcal{O}_{n_{1} n_{2} n_{3}}=\mathcal{O}_{n_{1}}^{\left(a_{1} a_{2}\right)} \mathcal{O}_{n_{2}}^{\left(a_{2} a_{3}\right)} \mathcal{O}_{n_{3}}^{\left(a_{3} a_{1}\right)}
$$

renormalized by triple ladders, and so on. It appears that in the large $N$ limit their scaling dimensions are additive, so that the spectrum of $\mathcal{O}_{n_{1} n_{2}}$ is $h_{1}+h_{2}$, the spectrum of $\mathcal{O}_{n_{1} n_{2} n_{3}}$ is $h_{1}+h_{2}+h_{3}$, etc., but we postpone a detailed study of the relevant Schwinger-Dyson equations. Here, $h_{i}$ are the eigenvalues which appear in the SYK spectrum; they are the solutions of (3.3). The picture of the 12-particle operator which is equivalent by the equation of motion to $\mathcal{O}_{0}^{\left(a_{1} a_{2}\right)} \mathcal{O}_{0}^{\left(a_{2} a_{3}\right)} \mathcal{O}_{0}^{\left(a_{3} a_{1}\right)}$, as well as the analogous operators 


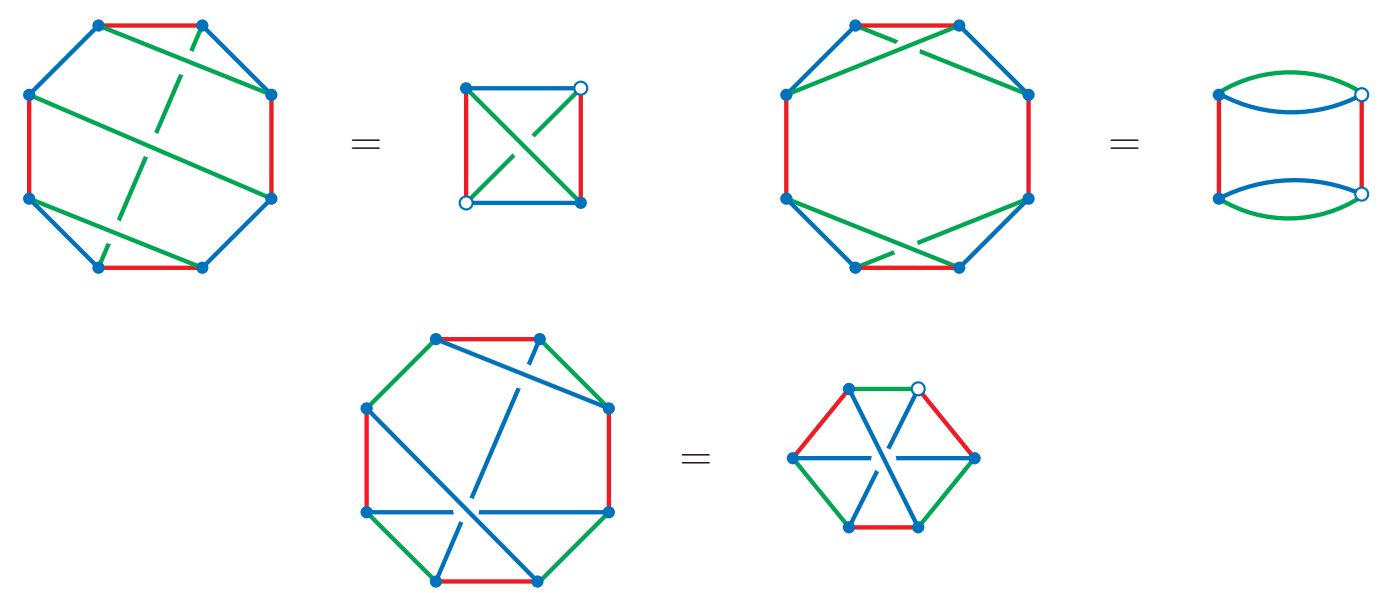

FIG. 11. The operators which can be represented as lower-order operators with derivative insertions shown by white dots.
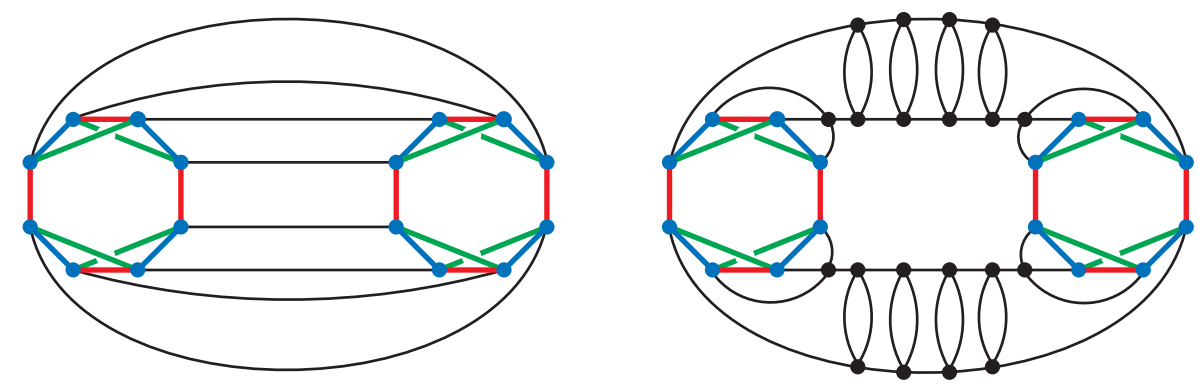

FIG. 12. An example of an operator renormalized by two ladder diagrams. The diagram with two ladders inserted (right) is of the same order as the diagram with operators connected directly (left). The black dots represent the tetrahedral coupling.

$\mathcal{O}_{0}^{\left(b_{1} b_{2}\right)} \mathcal{O}_{0}^{\left(b_{2} b_{3}\right)} \mathcal{O}_{0}^{\left(b_{3} b_{1}\right)}$ and $\mathcal{O}_{0}^{\left(c_{1} c_{2}\right)} \mathcal{O}_{0}^{\left(c_{2} c_{3}\right)} \mathcal{O}_{0}^{\left(c_{3} c_{1}\right)}$, is shown in Fig. 15.

We may construct additional operators renormalized by multiple ladders using the operators $\mathcal{O}_{n}^{\left[a_{1} a_{2}\right]}$, defined in (3.6), in addition to $\mathcal{O}_{n}^{\left(a_{1} a_{2}\right)}$. For example, there is a class of operators $\mathcal{O}_{n_{1}}^{\left[a_{1} a_{2}\right]} \mathcal{O}_{n_{2}}^{\left[a_{1} a_{2}\right]}$ of which the scaling dimensions appear to be $h_{1}+h_{2}$, where $h_{i}$ are the solutions of (3.7). Thus, the charges (2.6) and their products are not the only exceptions to the $m / 4$ rule (since the charges are conserved,

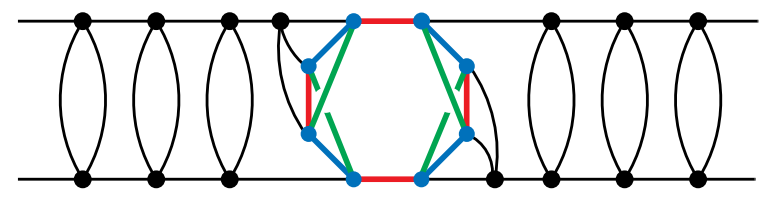

FIG. 13. A diagram with two ladders contributing to the correlation function $\left\langle\mathrm{O}_{8} \psi \psi \psi \psi \psi \psi\right\rangle$.

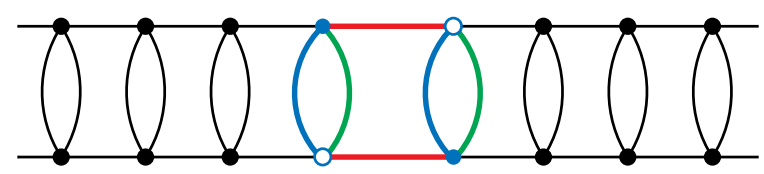

FIG. 14. Another representation for the same diagram. we a priori expect their scaling dimension to be zero). In fact, any operator of which the diagram contains a bubble subdiagram (i.e., two tensors with a double index contraction) is renormalized by a ladder, and there are as many ladders as there are bubbles. For example, a pillow operator contains two bubbles and is renormalized by two ladders.

Moreover, if we take an operator diagram renormalized by multiple ladders and change one vertex in the diagram from $\psi$ to $\partial_{t} \psi$ (blue to white vertex), it will still be renormalized by the same number of ladders. With derivatives, we can convert a pillow operator into the second operator in Fig. 8. It is easy to check that this operator is renormalized by two ladders. Since each of the ladders contains the $h=2$ zero mode in its spectrum, and a zero mode produces a low-temperature enhancement by a factor
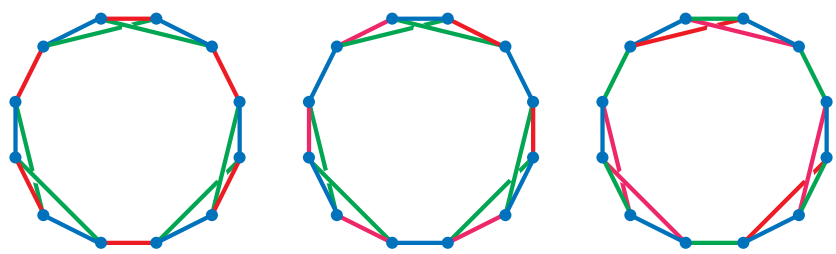

FIG. 15. Three 12-particle operators of the same topology, which are renormalized by three-ladder diagrams. 
of $\beta J$ [26], we expect the double ladder to produce an effect of order $(\beta J)^{2}$. The multiladder enhancements by $(\beta J)^{n}$ seem to be a new effect in the tensor model, which clearly needs to be studied in more detail.

To summarize, we find that:

(1) The operators containing bubble subgraphs are renormalized with as many ladder diagrams as there are bubble insertions.

(2) The operators obtained from operators with bubble subgraphs by inserting derivatives are renormalized by as many ladders as there were bubble insertions in the original diagram.

(3) The dimensions of operators which are renormalized with a single ladder are given by the solutions of the conformal kernel equation $g(h)=1$.

(4) The dimensions of the operators which are not renormalized by ladders are multiples of $1 / 4$.

These results are still far from providing the full information about the singlet spectrum of the $O(N)^{3}$ tensor quantum mechanics. In particular, we would like to have a more complete understanding of the operators renormalized by multiple ladders and to study their low-temperature contributions. We hope to address these questions elsewhere.

\section{SOME SCALING DIMENSIONS IN THE GURAU-WITTEN MODEL}

Let us now consider the $O(N)^{6}$ symmetric quantum mechanical model [17]. It contains four fermionic rank-3 tensors $\psi_{A}, A=0, \ldots 3$, each one transforming in the trifundamental representation under a different subset of the six $O(N)$ groups. The four fermionic tensors and the six $O(N)$ gauge groups may be visualized as the vertices and edges of a tetrahedron [17]. Thus, only two of the fermions transform under a given $O(N)$ symmetry. The GurauWitten Hamiltonian is

$$
H_{\mathrm{GW}}=-\frac{1}{4} g \psi_{0}^{a b c} \psi_{1}^{a d e} \psi_{2}^{f b e} \psi_{3}^{f d c} .
$$

The model contains bilinear operators of the form $O_{A}^{c_{1} c_{2}}=\psi_{A}^{a b c_{1}} \psi_{A}^{a b c_{2}}$. Let us focus on the operators with $A=0$ and 1 , which transform in the antisymmetric representation of the same $O(N)$ group and can mix with each other:

$$
\begin{aligned}
& O_{+}^{c_{1} c_{2}}=\psi_{0}^{a b c_{1}} \psi_{0}^{a b c_{2}}+\psi_{1}^{d e c_{1}} \psi_{1}^{d e c_{2}}, \\
& O_{-}^{c_{1} c_{2}}=\psi_{0}^{a b c_{1}} \psi_{0}^{a b c_{2}}-\psi_{1}^{d e c_{1}} \psi_{1}^{d e c_{2}} .
\end{aligned}
$$

The operator $O_{+}^{c_{1} c_{2}}$ is the charge of one of the six $O(N)$ symmetries; therefore, its scaling dimension vanishes. The operator $O_{-}^{c_{1} c_{2}}$ has another scaling dimension, $h_{-}$. The ladder diagrams contribute to the two-point function $\left\langle O_{-}^{c_{1} c_{2}}\left(t_{1}\right) O_{-}^{c_{3} c_{4}}\left(t_{2}\right)\right\rangle$, and we need to derive an appropriate Schwinger-Dyson equation. If we use $\psi_{0}^{a b c_{1}} \psi_{0}^{a b c_{2}}$ and $\psi_{1}^{d e c_{1}} \psi_{1}^{d e c_{2}}$ as the basis, then the kernel is a $2 \times 2$ symmetric matrix with zeros on the diagonal; hence, the two eigenvalues are equal and opposite. To fix the normalization, we note that the two functions $g_{ \pm}(h)$ are proportional to $\tilde{g}(h)$, which is given in (3.7). Therefore, $g_{+}(h)=\tilde{g}(h)$, and $g_{-}(h)=-\tilde{g}(h)$. The spectrum of solutions to $g_{+}(h)=1$ indeed includes $h=0$ corresponding to the conserved charge. The lowest solution to $g_{-}(h)=1$ is $h_{-} \approx 2.33$; this is the scaling dimension of operator $O_{-}^{c_{1} c_{2}}$. Thus, there are three quartic "pillow operators" made out of $\psi_{0}$ and $\psi_{1}$ : $O_{+}^{c_{1} c_{2}} O_{+}^{c_{1} c_{2}}$ of dimension $0, O_{+}^{c_{1} c_{2}} O_{-}^{c_{1} c_{2}}$ of dimension $h_{-}$, and $O_{-}^{c_{1} c_{2}} O_{-}^{c_{1} c_{2}}$ of dimension $2 h_{-}$. The third operator is the only pillow operator present in the gauged model where $O_{+}^{c_{1} c_{2}}$ is set to zero. Its dimension $2 h_{-} \approx 4.66$ makes it very irrelevant; we find six pillow operators with this dimension, corresponding to the presence of six different $O(N)$ groups.

We may also study the bilinear singlet operators like

$$
O_{-}^{n}=\psi_{0}^{a b c} \partial_{t}^{2 n+1} \psi_{0}^{a b c}-\psi_{1}^{d e c} \partial_{t}^{2 n+1} \psi_{1}^{d e c} .
$$

For $n=0$, this operator vanishes after the use of equations of motion, but it is nontrivial for $n=1,2, \ldots$. To calculate the scaling dimensions of these operators using the S-D equations, we note that the kernel is the SYK kernel,

$$
K_{\mathrm{SYK}}\left(t_{1}, t_{2} ; t_{3}, t_{4}\right)=-\frac{3}{4 \pi} \frac{\operatorname{sgn}\left(t_{1}-t_{3}\right) \operatorname{sgn}\left(t_{2}-t_{4}\right)}{\left|t_{1}-t_{3}\right|^{1 / 2}\left|t_{2}-t_{4}\right|^{1 / 2}\left|t_{3}-t_{4}\right|},
$$

times a $4 \times 4$ matrix with zeros on the diagonal and all the off-diagonal elements equal to the same value $B$. To determine $B$, we note that the kernel corresponding to the eigenvector $(1,1,1,1)$ with eigenvalue $3 B$ should exactly equal to the SYK kernel. This means that $B=1 / 3$, which gives the spectrum of the SYK model determined by $g(h)=1$ as given in equation (3.3). The three eigenvectors $(1,-1,0,0),(0,1,-1,0)$, and $(0,0,1,-1)$ have eigenvalue $-B=-1 / 3$; thus, the spectrum of corresponding operators is determined by

$$
-\frac{1}{3} g(h)=1
$$

The solutions to this equation are shown in Fig. $16 .{ }^{8}$ There is a series of solutions that lie slightly below $2 n+\frac{3}{2}$, for $n=1,2,3, \ldots$, and approach it at large $n$. In other words, they lie slightly below the naive dimensions of operators

\footnotetext{
${ }^{8}$ We may decompose the $O(N)^{6}$ invariant operators into irreducible representations of the symmetry group of the tetrahedron, which is isomorphic to $S_{4}$. Each solution to (6.6) corresponds to three operators belonging to a degree 3 representation of $S_{4}$.
} 


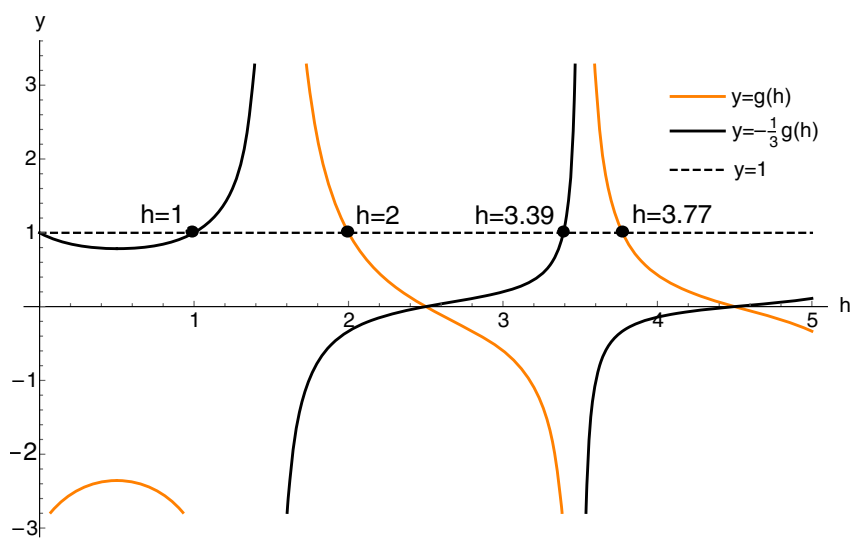

FIG. 16. Plot of the IR dimensions of the bilinear singlet operators in the GW model.

$O_{-}^{n}$. For $n=1$, the numerical value is 3.39 , which is close to 3.5. There is also an exact solution with $h=1$, the interpretation of which is not completely clear.

The dimensions of operators $O_{-}^{n}$ that we find are the same as in the Gross-Rosenhaus "generalized SYK model" [23] for $q=4$. In particular, the $h=1$ solution is present in that case as well, and the corresponding operator decouples. The Gross-Rosenhaus model that corresponds to the colored tensor model has $f=4$; i.e., it contains four flavors of Majorana fields, $\chi_{a}^{i}, a=1, \ldots, 4$. Its Hamiltonian may be written as

$$
H=J_{i j k l} \chi_{i}^{1} \chi_{j}^{2} \chi_{k}^{3} \chi_{l}^{4},
$$

where $J_{i j k l}$ are random couplings. The operators which are analogous to $O_{-}^{n}$ are $\chi_{i}^{1} \partial_{t}^{n+1} \chi_{i}^{1}-\chi_{j}^{2} \partial_{t}^{n+1} \chi_{j}^{2}$. The $n=0$ operator vanishes by the equation of motion for any value of $J_{i j k l}$, which appears to explain the decoupling of the $h=1$ mode.

\section{COUNTING SINGLET OPERATORS IN $\boldsymbol{d}=\mathbf{1}$}

In this section, we proceed to do the singlet operator counting in the $O(N)^{3}$ quantum mechanics more systematically. We employ the technique used in Refs. $[39,40]$ to find the partition function and free energy of gauge theory. In our case, we will see that the free energy diverges wildly, but nevertheless, this procedure allows us to count the operators in the gauged or ungauged fermionic and scalar theories.

We work in the one-dimensional spacetime with fields living in the trifundamental representation of $O(N)_{1} \times$ $O(N)_{2} \times O(N)_{3}$, in the limit of $N \rightarrow \infty$. We will mainly address the case of the free tensor model, which describes the UV fixed point, but also make comments about the IR theory. The partition function may be written in the form

$$
\mathcal{Z}=\sum_{\mathcal{O}_{i}} x^{h_{i}}, \quad x \equiv e^{-\beta},
$$

where $\mathcal{O}_{i}$ are all operators in the theory which are singlets under $O(N)^{3}$. Here, $h_{i}$ are the conformal dimensions, so in the UV, this partition function is

$$
\mathcal{Z}=\sum_{k} n_{k} x^{k h_{\mathrm{Uv}}}
$$

where $k$ is the number of fields comprising an operator and $n_{k}$ is the number of admissible operators for each $k$. In what follows, we call $k$ the order of an operator. For the fermionic model, $h_{\mathrm{UV}}=(d-1) / 2$, and for bosonic, it is $(d-2) / 2$.

The partition function counts all operators including the disconnected ones. To restrict ourselves exclusively to the connected operators, we have to compute the single-sum partition function defined as

$$
\log \mathcal{Z}(x)=\sum_{m=1}^{\infty} \frac{1}{m} \mathcal{Z}_{\text {s.s. }}\left(x^{m}\right)
$$

To find $\mathcal{Z}_{\text {s.s. }}$ explicitly, we use an elegant formula from Ref. [40]:

$$
\mathcal{Z}_{\text {s.s. }}(x)=\log \mathcal{Z}(x)+\sum_{m \in \Omega}(-1)^{\nu_{m}} \frac{1}{m} \log \mathcal{Z}\left(x^{m}\right) .
$$

Here, $m$ belongs to the set of square-free integers $\Omega=\{2,3,5,6,7,10,11,13, \ldots\}$ :

$$
m=\prod_{i=1}^{\nu_{m}} p_{i}, \quad p_{i} \text { prime. }
$$

Our goal in this section is to find the single-sum partition function for the scalar and fermionic tensor models. The partition function for the scalar theory in the UV with one group can be found as [37-39]

$$
\mathcal{Z}^{S}=\int d M \exp \left(\sum_{m=1}^{\infty} \frac{1}{m} z_{S, d}\left(x^{m}\right) \chi\left(M^{m}\right)\right),
$$

and for the fermionic theory, it is

$$
\mathcal{Z}^{F}=\int d M \exp \left(\sum_{m=1}^{\infty} \frac{(-1)^{m+1}}{m} z_{F, d}\left(x^{m}\right) \chi\left(M^{m}\right)\right),
$$

with $M$ in the symmetry group and $\chi(M)$ being the character of the desired representation. In our case, we substitute

$$
\begin{aligned}
& M \rightarrow M_{1} M_{2} M_{3}, \quad \chi(M) \rightarrow \chi\left(M_{1}\right) \chi\left(M_{2}\right) \chi\left(M_{3}\right), \\
& \qquad M_{i} \in O(N)_{i} \\
& \text { and take } \chi(M)=\operatorname{tr} M
\end{aligned}
$$


The single-letter partition functions for scalars and (Majorana) fermions correspondingly are as follows:

$$
\begin{aligned}
& z_{S, d}(x)=\frac{x^{\frac{d}{2}-1}(1+x)}{(1-x)^{d-1}}, \\
& z_{F, d}(x)=\frac{2^{\left.2 \frac{d}{2}\right\rfloor} x^{\frac{d-1}{2}}}{(1-x)^{d-1}} .
\end{aligned}
$$

To find $\mathcal{Z}$, we will need the integrals of characters of $O(N)$ [40]:

$$
\begin{aligned}
& \int d M \prod_{l}\left(\operatorname{tr} M^{l}\right)^{a_{l}} \\
& =\prod_{l}\left[\begin{array}{cc}
l \text { odd } a_{l} \text { even } & (2 l)^{a_{l} / 2} \frac{1}{\sqrt{\pi}} \Gamma\left(\frac{a_{l}}{2}+\frac{1}{2}\right), \\
l \text { even } & \sum_{k=0}^{a_{l} / 2}\left(\begin{array}{c}
a_{l} \\
2 k
\end{array}\right)(2 l)^{k} \frac{1}{\sqrt{\pi}} \Gamma\left(k+\frac{1}{2}\right) .
\end{array}\right.
\end{aligned}
$$

In the next chapter, we first find partition functions for both the fermionic and scalar $d=1$ models without the constraint that the charges (2.6) vanish. Then, to find the partition function for the operators in the gauged model, we subtract the contribution from the operators containing $O(N)$ charge, or a "bubble" subdiagram (2.6) (see Fig. 3). Such operators should vanish in the gauged version of quantum mechanics.

\section{A. Fermions}

The single-letter partition function for real fermions $z_{F, d}$ is not well defined in one dimension. This reflects the divergence of the partition function (and hence free energy). To regularize it, we formally proceed in $(1+2 \epsilon)$ dimension and neglect all the terms proportional to $\epsilon$ in the single-letter partition function; in other words, we simply take

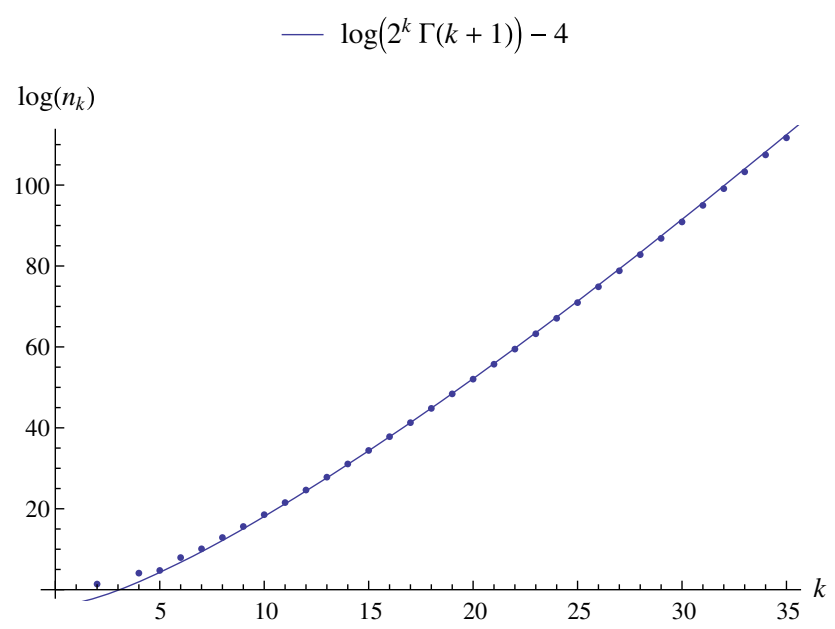

$$
z_{F, 1+2 \epsilon}=x^{\epsilon}
$$

We can justify this choice as follows. The single-letter partition function counts all local operators containing one field $\psi^{a b c}$ with any number of derivatives. In our case, the only such operator is $\psi^{a b c}$; since $\partial_{t} \psi^{a b c}$ vanishes by equations of motion in the free theory, all the operators with higher derivatives will vanish, too.

In other words, in the fermionic case, we are counting only the operators made of fermions without derivatives. We can think of this as operator counting in a $d=0$ model (for a review, see Ref. [2]), but with the Fermi statistics imposed.

Computing $\mathcal{Z}$ and using (7.7) and (7.11), we find to the first several orders in $x$

$$
\begin{aligned}
\mathcal{Z}^{F}= & 1+4 x^{4 \epsilon}+70 x^{8 \epsilon}+116 x^{10 \epsilon}+3062 x^{12 \epsilon} \\
& +24788 x^{14 \epsilon}+409869 x^{16 \epsilon}+\cdots
\end{aligned}
$$

From this, we can find the single-sum partition function, which counts connected operators:

$$
\begin{aligned}
\mathcal{Z}_{\text {s.s. }}^{F}= & 4 x^{4 \epsilon}+60 x^{8 \epsilon}+116 x^{10 \epsilon}+2802 x^{12 \epsilon}+24324 x^{14 \epsilon} \\
& +396196 x^{16 \epsilon}+\cdots .
\end{aligned}
$$

The order $2 k$ in $x^{2 k e}$ gives the number of fermions in the operator. So, we see there are four four-fermion operators: one tetrahedron and three differently colored pillows (see Fig. 4). Note that, although we employed a gauged theory to count these operators, the pillows and other operators containing $O(N)$ charges are still present. At the sixth order, there are no operators because of the Fermi statistics as we noticed before, but at order 8 there are 60 operators.

The number of $2 k$-particle operators grows roughly as (see Fig. 17):

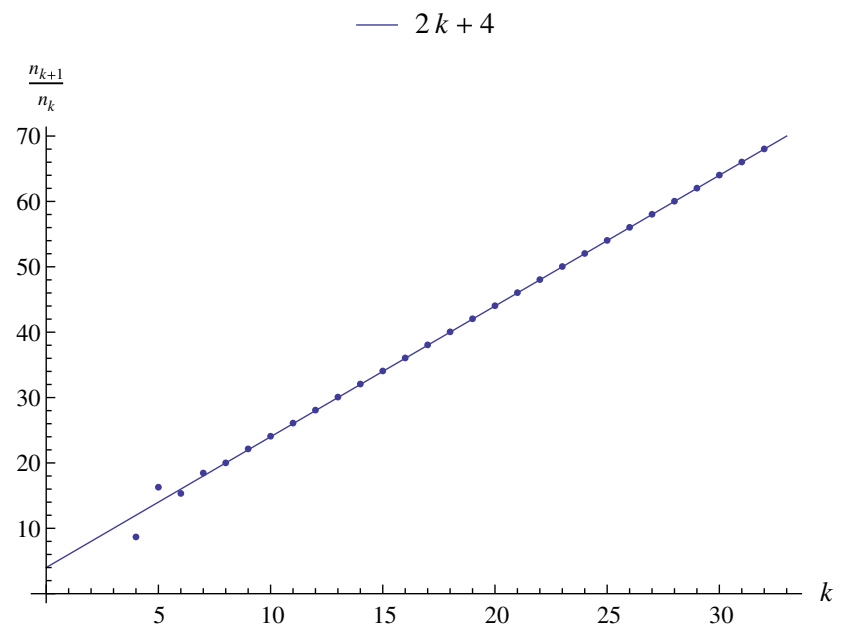

FIG. 17. Logarithm of the number of allowed $(2 k)$-particle fermionic operators as a function of $k$. We see that the number of operators grows like $\sim k ! 2^{k}$. 


$$
n_{2 k} \sim 2^{k} k !
$$

To count operators in the gauged model where the vanishing of $O(N)$ charges (2.6) is imposed, we have to disregard the operators containing their insertions, i.e., the bubble subgraphs. In order to do that, we subtract the operators having the same quantum numbers as a bubble in the exponent of (7.7). Each $O(N)$ charge (2.6) is antisymmetric in its two indices, which means that it lives in the representation $(N \otimes N)_{\text {antisym }}$ with the character:

$$
\chi_{A}(M) \equiv \chi_{(N \otimes N)_{\text {antisym }}}(M)=\frac{1}{2}\left((\operatorname{tr} M)^{2}-\operatorname{tr} M^{2}\right) .
$$

The bubble is a bosonic operator, and its conformal dimension in the UV is $2 \epsilon$. Bringing it all together, we find that the partition function for operators in the gauge theory is

$$
\begin{aligned}
\mathcal{Z}^{F(\text { gauge })}= & \int d M_{1} d M_{2} d M_{3} \\
& \times \exp \left(\sum _ { m = 1 } ^ { \infty } \frac { 1 } { m } \left((-1)^{m+1} x^{m \epsilon} \chi\left(M_{1}\right) \chi\left(M_{2}\right) \chi\left(M_{3}\right)\right.\right. \\
& \left.\left.-x^{2 m \epsilon}\left(\chi_{A}\left(M_{1}\right)+\chi_{A}\left(M_{2}\right)+\chi_{A}\left(M_{3}\right)\right)\right)\right) .
\end{aligned}
$$

The single-sum partition function for the gauge theory then is as follows:

$$
\begin{gathered}
\mathcal{Z}_{\text {s.s. }}^{F(\text { gauge })}=x^{4 \epsilon}+17 x^{8 \epsilon}+24 x^{10 \epsilon}+617 x^{12 \epsilon}+4887 x^{14 \epsilon} \\
+82466 x^{16 \epsilon}+\cdots \\
-\log \left(2^{k} \Gamma(k+1)\right)-2.82
\end{gathered}
$$

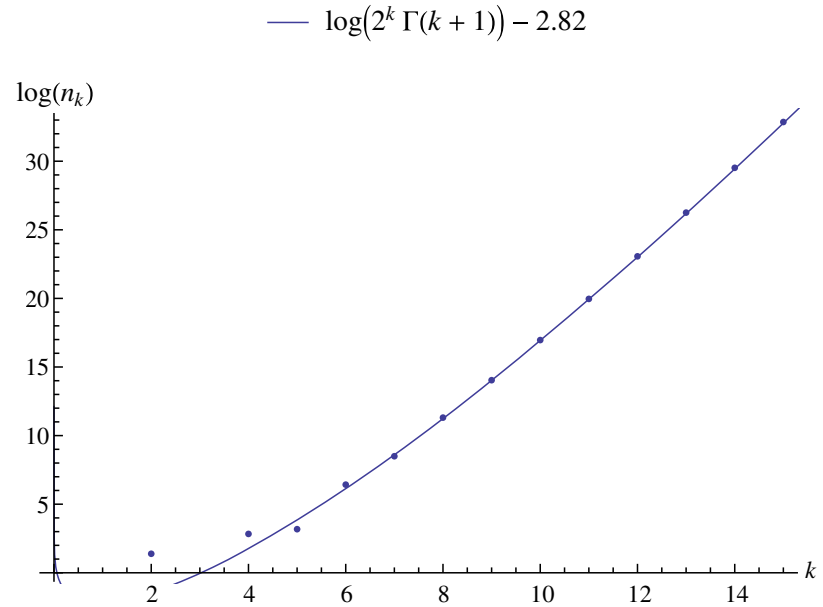

We see that at the fourth order we are left with one operator, namely, the tetrahedron. At the eighth order, we see 17 operators, as we already found in Sec. IVA via explicit construction (see Fig. 9). We have computed the single-sum partition function up to order 30, and the result matches the same factorial growth as in the model where the $O(N)^{3}$ symmetry is not gauged (see Fig. 18).

Finally, let us comment on the IR theory, where we believe there is similarly rapid growth of the number of operators as a function of the conformal dimension. Since for the majority of $2 k$-particle operators the large $N$ IR dimension is $h=k / 2$, in view of the result (7.15), we expect the number of operators of dimension $h$ to grow as $\Gamma(2 h+1)$, up to an exponential prefactor.

\section{B. Bosons}

We can also count the allowed operators in the scalar theory. Proceeding in the same fashion, we define singleletter partition function in $(1+2 \epsilon)$ dimensions as follows,

$$
z_{S, 1+2 \epsilon}=x^{-\frac{1}{2}+\epsilon}(1+x)
$$

where $-\frac{1}{2}+\epsilon$ is the dimension of the scalar field. The partition function is

$$
\begin{aligned}
\mathcal{Z}^{S}= & 1+x^{2 \epsilon}\left(x^{-1}+1+x\right) \\
& +x^{4 \epsilon}\left(5 x^{-2}+5 x^{-1}+14+5 x+5 x^{2}\right) \\
& +x^{6 \epsilon}\left(16 x^{-3}+34 x^{-2}+101 x^{-1}+108\right. \\
& \left.+101 x+34 x^{2}+16 x^{3}\right)+\cdots .
\end{aligned}
$$

The single-sum partition function, which includes the operators with bubble insertions, is

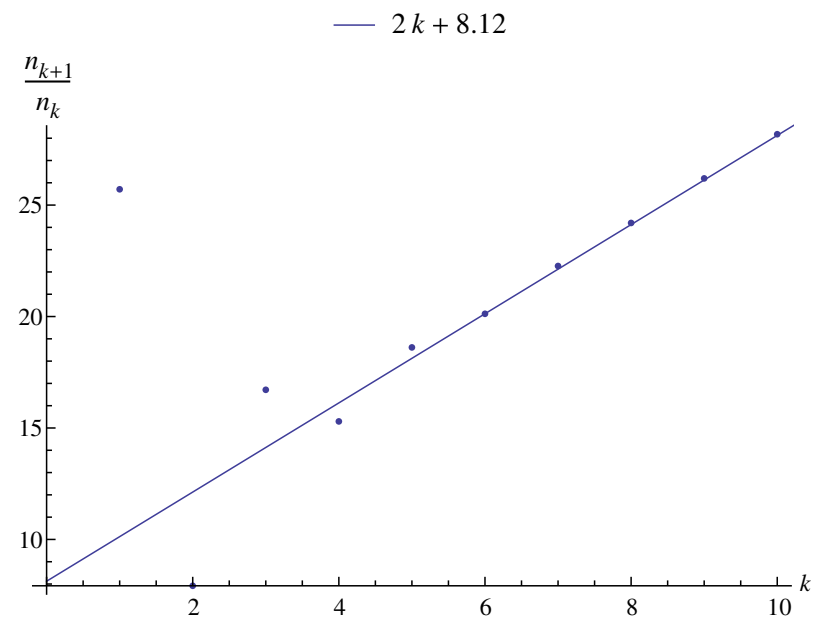

FIG. 18. Left: The logarithm of the number of $(2 k)$-particle operators $n_{2 k}$ in the model where $O(N)^{3}$ symmetry is gauged. The asymptotic of the number of operators is roughly the same as in the ungauged theory. Right: the ratio $n_{2 k+2} / n_{2 k}$ plotted against $k$. The linear behavior clearly indicates $\sim 2^{k} k$ ! growth. 


$$
\begin{aligned}
\mathcal{Z}_{\text {s.s. }}^{S}= & x^{2 \epsilon}\left(x^{-1}+1+x\right) \\
& +x^{4 \epsilon}\left(4 x^{-2}+4 x^{-1}+12+4 x+4 x^{2}\right) \\
& +x^{6 \epsilon}\left(11 x^{-3}+25 x^{-2}+79 x^{-1}+86+79 x\right. \\
& \left.+25 x^{2}+11 x^{3}\right)+\cdots .
\end{aligned}
$$

In the second order, we have operators $\phi^{a b c} \phi^{a b c}$, $\phi^{a b c} \partial_{t} \phi^{a b c}$, and $\partial_{t} \phi^{a b c} \partial_{t} \phi^{a b c}$. In the fourth order, we find the pillows and tetrahedra with various insertions of $\partial_{t}$. This partition function also diverges at $\epsilon \rightarrow 0$ and displays the factorial growth of the number of operators with their order.

To count operators in the gauged theory, we once again have to take care of the subgraphs corresponding to the gauge group charge. For a scalar theory, the gauge charge operator is

$$
Q^{a_{1} a_{2}}=\phi^{a_{1} b c} \stackrel{\leftrightarrow}{\partial_{t}} \phi^{a_{2} b c}
$$

This operator lives in the adjoint representation, just like the gauge field. Its dimension is $2 \epsilon=\left(-\frac{1}{2}+\epsilon\right)+\left(\frac{1}{2}+\epsilon\right)$. The character of the adjoint representation is

$$
\chi_{\text {adj }}(M)=\frac{1}{2}\left((\operatorname{tr} M)^{2}-\operatorname{tr} M^{2}\right) .
$$

Taking all this into account, we write the partition function as

$$
\begin{aligned}
\mathcal{Z}^{S(\text { gauge })}= & \int d M_{1} d M_{2} d M_{3} \times \exp \left(\sum _ { m = 1 } ^ { \infty } \frac { 1 } { m } \left(\left(x^{-\frac{m}{2}+\epsilon m}+x^{\frac{m}{2}+\epsilon m}\right) \chi\left(M_{1}\right) \chi\left(M_{2}\right) \chi\left(M_{3}\right)\right.\right. \\
& \left.\left.-\chi_{\text {adj }}\left(M_{1}\right) x^{2 m \epsilon}-\chi_{\text {adj }}\left(M_{2}\right) x^{2 m \epsilon}-\chi_{\text {adj }}\left(M_{3}\right) x^{2 m \epsilon}\right)\right)
\end{aligned}
$$

To the first six orders, the partition function reads as

$$
\begin{aligned}
\mathcal{Z}^{S(\text { gauge })}= & 1+x^{2 \epsilon}\left(x^{-1}+1+x\right) \\
& +x^{4 \epsilon}\left(5 x^{-2}+5 x^{-1}+11+5 x+5 x^{2}\right) \\
& +x^{6 \epsilon}\left(16 x^{-3}+34 x^{-2}+77 x^{-1}+84\right. \\
& \left.+77 x+34 x^{2}+16 x^{3}\right)+\cdots
\end{aligned}
$$

The single-sum partition function, which counts only the operators with connected diagrams, is as follows

$$
\begin{aligned}
\mathcal{Z}_{\text {s. }}^{S \text { (gauge })}= & x^{2 \epsilon}\left(x^{-1}+1+x\right) \\
& +x^{4 \epsilon}\left(4 x^{-2}+4 x^{-1}+9+4 x+4 x^{2}\right) \\
& +x^{6 \epsilon}\left(11 x^{-3}+25 x^{-2}+58 x^{-1}+65\right. \\
& \left.+58 x+25 x^{2}+11 x^{3}\right)+\cdots
\end{aligned}
$$

The first term in this expression corresponds to the operators $\phi^{a b c} \phi^{a b c}, \phi^{a b c} \partial_{t} \phi^{a b c}$, and $\partial_{t} \phi^{a b c} \partial_{t} \phi^{a b c}$ (the second of these operators is a total derivative; such descendant operators are included in the counting). The number 11 in the third term corresponds to all the sixparticle graphs discussed in Sec. IV. Now, the number of operators containing a string of $2 k$ scalars is approximately

$$
n_{2 k} \sim 2^{2 k} \times 2^{k} k !
$$

Compared to the fermionic case (7.15), we have an additional factor of $2^{2 k}$. As we will see in the next section, for $d=0$, the leading asymptotic for the number of operators is the same for scalars and fermions. Therefore, the factor
$2^{2 k}$ comes from distributing the time derivatives $\partial_{t}$ among $2 k$ fields. Since in the free theory $\partial_{t}^{2} \phi^{a b c}=0$, each of the $2 k$ fields may be acted on by one or no derivatives. This indeed contributes a factor of $2^{2 k}$.

\section{COUNTING THE INVARIANTS IN $\boldsymbol{d}=\mathbf{0}$}

Here, we use methods similar to those in the previous section to discuss the counting of invariants in the $d=0$ model, which is simply an integral over the tensor. The construction and counting of such invariants, which are made out of products of tensors with all indices contracted, has been addressed in Refs. [2,42-46]. These papers primarily discuss the complex bosonic rank- $r$ tensor models which possess $U(N)^{r}$ symmetry. We will first consider the bosonic rank-3 tensor model with $O(N)^{3}$ symmetry and perform the counting using the methods developed in Refs. [39,40]. The model of a real fermionic tensor $\psi^{a b c}$ does not work in $d=0$; since the $O(N)^{3}$ invariant $\psi^{a b c} \psi^{a b c}$ vanishes, it is impossible to write down a Gaussian integral. One can write down models of complex fermionic tensors in $d=0$, but we will not study them here. We will address the bosonic rank-3 symmetric traceless and antisymmetric tensors in Sec. VIII A and the bosonic complex tensors with $U(N)^{3}$ and $U(N)^{2} \times O(N)$ symmetries in Sec. VIII B.

The single-letter partition function counts all the invariants containing one field. In our case, the only such operator is $\phi^{a b c}$, so the single-letter partition function is

$$
z_{S, 0}(x)=x .
$$


$\log \left(2^{k} \Gamma(k+1)\right)-0.46$

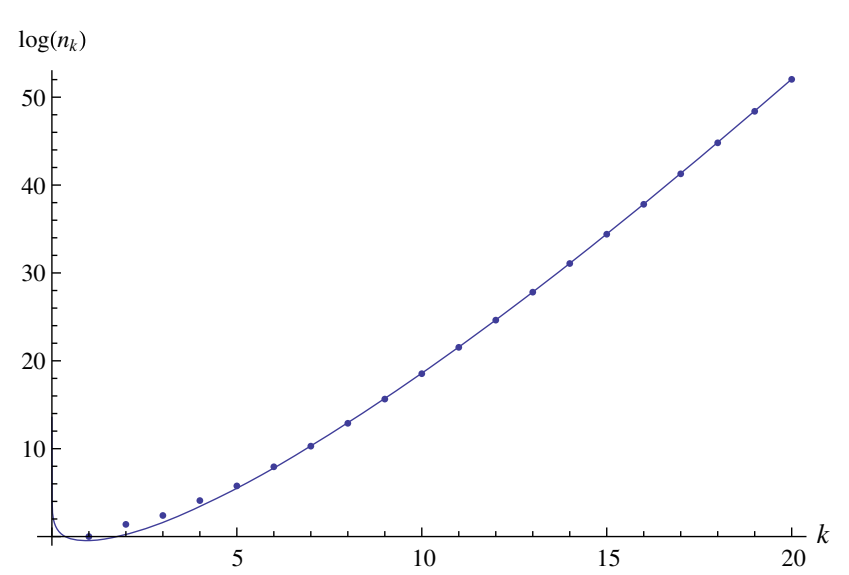

$-2 k+0.04$

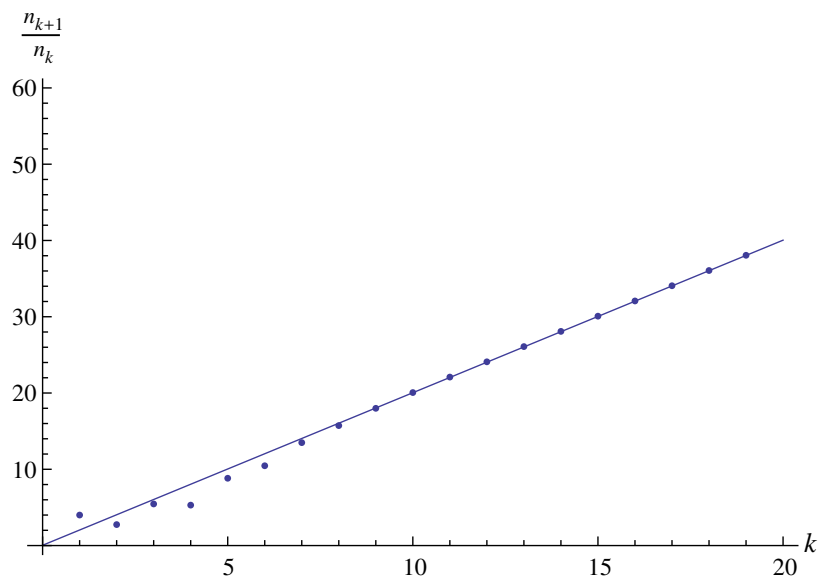

FIG. 19. Logarithm of the number of invariants with $2 k$ scalars as a function of $k$. The number grows as $\sim k ! 2^{k}$.

The invariants in this case are given by the diagrams with $2 k$ vertices and three edges of different colors meeting at each vertex. Thus, the invariants are isomorphic to the Feynman diagrams in the theory of three scalar fields with interaction $\varphi_{1} \varphi_{2} \varphi_{3}$. Every edge of the diagram is assigned one of the three colors, and every vertex joins the edges of three different colors. This is a nontrivial condition; for example, one-particle reducible graphs cannot be colored in this way. We consider different colorings of the diagrams as different invariants, so each topology can enter multiple times if there are several distinct ways to color it.

Using (7.7), we find the full partition function,

$$
\begin{aligned}
\mathcal{Z}^{0}= & \int d M_{1} d M_{2} d M_{3} \\
& \times \exp \left(\sum_{m=1}^{\infty} \frac{1}{m} x^{m} \chi\left(M_{1}^{m}\right) \chi\left(M_{2}^{m}\right) \chi\left(M_{3}^{m}\right)\right),
\end{aligned}
$$

where we have used the character of a trifundamental representation (7.8). Taking this integral and using (7.11), we find in the first several orders

$$
\begin{aligned}
\mathcal{Z}^{0}= & 1+x^{2}+5 x^{4}+16 x^{6}+86 x^{8}+448 x^{10}+3580 x^{12} \\
& +34981 x^{14}+\cdots
\end{aligned}
$$

This partition function counts all the invariants, including the disconnected ones. To remove the latter, we compute the single-sum partition function using (7.4):

$$
\begin{aligned}
\mathcal{Z}_{\text {s.s. }}^{0}= & x^{2}+4 x^{4}+11 x^{6}+60 x^{8}+318 x^{10}+2806 x^{12} \\
& +29359 x^{14}+\cdots .
\end{aligned}
$$

The only two-scalar invariant is $\phi^{a b c} \phi^{a b c}$. The four fourscalar invariants are the three inequivalent pillows and the tetrahedron, shown in Fig. 4. The 11 six-scalar invariants are the ones shown in Fig. 5.
The number of invariants made out of $2 k$ fields grows asymptotically as (see Fig. 19):

$$
n_{2 k} \sim 2^{k} k !
$$

We can find this asymptotic from an analytic estimate. The key observation is that the integral (7.11) grows factorially as $\left(a_{l} / 2\right)$ ! for large $a_{l}$, while only as a power $l^{a_{l} / 2}$ for large $l$. Besides, for large $a_{l}$, there is no difference in the leading order between odd and even $l$. Therefore, the leading contribution to $x^{2 k}$ will come simply from the $m=1$ term:

$$
\begin{aligned}
n_{2 k} & \sim \frac{1}{(2 k) !} \int d M_{1} d M_{2} d M_{3}\left(\chi\left(M_{1}\right) \chi\left(M_{2}\right) \chi\left(M_{3}\right)\right)^{2 k} \\
& =\frac{1}{(2 k) !}\left(2^{k} \Gamma(k+1 / 2)\right)^{3} \sim 2^{k} k !
\end{aligned}
$$

Since the dominant term originates only from $m=1$ term, the same estimate is valid for the fermions.

\section{A. Symmetric traceless and antisymmetric tensors}

Let us also discuss the counting of invariants in models with a single $O(N)$ symmetry, where we will consider the tensors which are either symmetric traceless or fully antisymmetric. Such models with the tetrahedral interactions were recently studied in Ref. [12], where evidence was provided that they have melonic large $N$ limits. The full partition function is

$$
\mathcal{Z}=\int d M \exp \left(\sum_{m=1}^{\infty} \frac{1}{m} x^{m} \chi\left(M^{m}\right)\right)
$$

where for the three-index symmetric traceless representation the character in the large $N$ limit is ${ }^{9}$

\footnotetext{
${ }^{9}$ The more complicated expression at finite $N$ may be extracted from Eq. (2.4) of Ref. [12].
} 
$\chi^{+}(M)=\frac{1}{6}(\operatorname{tr} M)^{3}+\frac{1}{2} \operatorname{tr} M \operatorname{tr} M^{2}+\frac{1}{3} \operatorname{tr} M^{3}-\operatorname{tr} M$.

For the fully antisymmetric representation, the character is

$$
\chi^{-}(M)=\frac{1}{6}(\operatorname{tr} M)^{3}-\frac{1}{2} \operatorname{tr} M \operatorname{tr} M^{2}+\frac{1}{3} \operatorname{tr} M^{3} .
$$

In the symmetric traceless case, the partition function is found to be

$$
\mathcal{Z}^{+}=1+x^{2}+3 x^{4}+9 x^{6}+32 x^{8}+135 x^{10}+709 x^{12}+\cdots .
$$

Extracting the single-sum expression, we find

$$
\mathcal{Z}_{\text {s.s. }}^{+}=x^{2}+2 x^{4}+6 x^{6}+20 x^{8}+91 x^{10}+509 x^{12}+\cdots .
$$

The numbers of $O(N)$ invariants made of $2 k$ fields are the same as the numbers of connected tadpole-free vacuum diagrams in the $\phi^{3}$ theory (here, the edges have only one color). They are smaller than the corresponding numbers in (8.4) referring to the $O(N)^{3}$ theory. For example, at order 4, we now have only two distinct invariants; in addition to the tetrahedron, there is only one pillow, since there are no distinct colorings of it. For large $k$, the number of invariants can be estimated similarly to the trifundamental case (8.6). Once again, the term with $m=1$ dominates. Moreover, out of the four terms in (8.8), $(\operatorname{tr} M)^{3} / 6$ gives the biggest contribution. Therefore,

$$
n_{2 k}^{ \pm} \sim \frac{1}{(2 k) ! 6^{2 k}} \int d M(\operatorname{tr} M)^{6 k} \sim\left(\frac{3}{2}\right)^{k} k !
$$

where we used the integrals (7.11).

Since $(\operatorname{tr} M)^{3} / 6$ dominates, the same asymptotic formula is valid for the three-index antisymmetric case. Here, the partition function is found to be

$$
\mathcal{Z}^{-}=1+x^{2}+3 x^{4}+7 x^{6}+24 x^{8}+86 x^{10}+426 x^{12}+\cdots,
$$

and the single-sum partition function is

$\mathcal{Z}_{\text {s.s. }}^{-}=x^{2}+2 x^{4}+4 x^{6}+14 x^{8}+54 x^{10}+298 x^{12}+\cdots$.

\section{B. Complex 3-tensors}

Let us now consider the complex 3-tensors with $U(N)^{3}$ or $U(N)^{2} \times O(N)$ symmetries. The latter symmetry is particularly interesting because it is preserved by the tetrahedral interaction $\phi^{a_{1} b_{1} c_{1}} \bar{\phi}^{a_{1} b_{2} c_{2}} \phi^{a_{2} b_{1} c_{2}} \bar{\phi}^{a_{2} b_{2} c_{1}}$. This means that there are interacting melonic theories with the $U(N)^{2} \times O(N)$ symmetry $[6,9,18]$.

In the $U(N)^{3}$ case, we have the fields $\phi^{a b c}$ and $\bar{\phi}^{a b c}$, which are in the trifundamental representations $N \times N \times N$ and $\bar{N} \times \bar{N} \times \bar{N}$ respectively. The partition function reads

$$
\mathcal{Z}^{U(N)^{3}}=\int d M_{1} d M_{2} d M_{3} \exp \left(\sum_{m=1}^{\infty} \frac{z\left(x^{m}\right)}{m}\left(\chi\left(M_{1}^{m}\right) \chi\left(M_{2}^{m}\right) \chi\left(M_{3}^{m}\right)+\bar{\chi}\left(M_{1}^{m}\right) \bar{\chi}\left(M_{2}^{m}\right) \bar{\chi}\left(M_{3}^{m}\right)\right)\right) .
$$

It is straightforward to compute it using the following large $N$ result [40]:

$$
\int d M \prod_{l \geq 1}\left(\operatorname{tr} M^{l}\right)^{a_{l}}\left(\operatorname{tr} \bar{M}^{l}\right)^{b_{l}}=\prod_{l \geq 1} l^{a_{l}} a_{l} ! \delta_{a_{l}, b_{l}} .
$$

For the scalar, we take $z_{S, 0}(x)=x$ and find

$$
\mathcal{Z}^{U(N)^{3}}=1+x^{2}+4 x^{4}+11 x^{6}+43 x^{8}+161 x^{10}+\cdots .
$$

This expansion matches the results obtained in Ref. [46] using group-theoretic methods. Extracting from $\mathcal{Z}$ the single-sum partition function, we find

$$
\mathcal{Z}_{\text {s.s. }}^{U(N)^{3}}=x^{2}+3 x^{4}+7 x^{6}+26 x^{8}+97 x^{10}+\cdots .
$$

The coefficient 3 of $x^{4}$ is in agreement with the fact that the tetrahedron invariant is not allowed by the $U(N)^{3}$ symmetry. Only the three pillow invariants are allowed, and their form is

$$
\begin{aligned}
& \phi^{a_{1} b_{1} c_{1}} \overline{\boldsymbol{\phi}}^{a_{1} b_{1} c_{2}} \boldsymbol{\phi}^{a_{2} b_{2} c_{2}} \overline{\boldsymbol{\phi}}^{a_{2} b_{2} c_{1}}, \\
& \boldsymbol{\phi}^{a_{1} b_{1} c_{1}} \overline{\boldsymbol{\phi}}^{a_{1} b_{2} c_{1}} \boldsymbol{\phi}^{a_{2} b_{2} c_{2}} \overline{\boldsymbol{\phi}}^{a_{2} b_{1} c_{2}}, \\
& \boldsymbol{\phi}^{a_{1} b_{1} c_{1}} \overline{\boldsymbol{\phi}}^{a_{2} b_{1} c_{1}} \boldsymbol{\phi}^{a_{2} b_{2} c_{2}} \overline{\boldsymbol{\phi}}^{a_{1} b_{2} c_{2}} .
\end{aligned}
$$

The asymptotic number of operators can be estimated as follows. As in the $O(N)$ case, the integral (8.16) grows factorially in $a_{l}$ and only as a power in $l$. It means that the term with $m=1$ again dominates. Besides, to get a nonzero answer, we need to extract the term with an equal number of $\chi\left(M_{i}\right)$ and $\bar{\chi}\left(M_{i}\right)$. Therefore, 
$n_{2 k}^{U(N)^{3}} \sim\left(\begin{array}{c}2 k \\ k\end{array}\right) \frac{1}{(2 k) !} \int d M_{1} d M_{2} d M_{3} \prod_{i=1}^{3} \chi\left(M_{i}\right)^{k} \bar{\chi}\left(M_{i}\right)^{k} \sim k !$

In the $U(N)^{2} \times O(N)$ case, we have representations $N \times N \times N$ and $\bar{N} \times \bar{N} \times N$, so that

$$
\begin{aligned}
& \mathcal{Z}^{U(N)^{2} \times O(N)} \\
& =\int d M_{1} d M_{2} d M_{3} \exp \left(\sum _ { m = 1 } ^ { \infty } \frac { z ( x ^ { m } ) } { m } \left(\chi\left(M_{1}^{m}\right) \chi\left(M_{2}^{m}\right)\right.\right. \\
& \left.\left.\quad+\bar{\chi}\left(M_{1}^{m}\right) \bar{\chi}\left(M_{2}^{m}\right)\right) \chi\left(M_{3}^{m}\right)\right),
\end{aligned}
$$

where the matrices $M_{1}, M_{2}$ belong to $U(N)$, while $M_{3}$ belongs to $O(N)$. The scalar partition function has the following expansion:

$$
\begin{aligned}
\mathcal{Z}^{U(N)^{2} \times O(N)}= & 1+x^{2}+6 x^{4}+21 x^{6}+147 x^{8} \\
& +1043 x^{10}+\cdots .
\end{aligned}
$$

Extracting the single-sum partition function, we find

$$
\mathcal{Z}_{\text {s.S. }}^{U(N)^{2} \times O(N)}=x^{2}+5 x^{4}+15 x^{6}+111 x^{8}+821 x^{10}+\cdots .
$$

The coefficient 5 of $x^{4}$ is in agreement with the fact that, in addition to the tetrahedron invariant, there are four pillow invariants allowed by the $U(N)^{2} \times O(N)$ symmetry:

$$
\begin{array}{ll}
\phi^{a_{1} b_{1} c_{1}} \bar{\phi}^{a_{1} b_{1} c_{2}} \phi^{a_{2} b_{2} c_{1}} \bar{\phi}^{a_{2} b_{2} c_{2}}, & \phi^{a_{1} b_{1} c_{1}} \bar{\phi}^{a_{1} b_{1} c_{2}} \phi^{a_{2} b_{2} c_{2}} \bar{\phi}^{a_{2} b_{2} c_{1}}, \\
\phi^{a_{1} b_{1} c_{1}} \bar{\phi}^{a_{1} b_{2} c_{1}} \phi^{a_{2} b_{2} c_{2}} \bar{\phi}^{a_{2} b_{1} c_{2}}, & \phi^{a_{1} b_{1} c_{1}} \bar{\phi}^{a_{2} b_{1} c_{1}} \phi^{a_{2} b_{2} c_{2}} \bar{\phi}^{a_{1} b_{2} c_{2}} .
\end{array}
$$

Using the same method as in the $U(N)^{3}$ case, the asymptotic growth can be found to be

$$
n_{2 k}^{U(N)^{2} \times O(N)} \sim 2^{k} k !
$$

\section{HAGEDORN TRANSITION}

The special features of the thermodynamics of free theories where the fields are tensors of rank $r \geq 3$ under some global symmetry group were recently studied in Ref. [40]. It was found that the Hagedorn temperature vanishes in the large $N$ limit as $\sim 1 / \log N$ [40]. In this section, we show that this also applies to the models with $O(N)^{3}$ symmetry studied in this paper.

An essential feature of the large $N$ tensor models is that the low-temperature expansion of the partition function has the approximate structure $\sum_{k} 2^{k} k ! x^{2 k}$, where $-\ln x$ is proportional to $\beta$. This power series is divergent and non-Borel summable; therefore, strictly speaking, the partition function is not defined for any finite temperature. To illustrate the basic points, we study the large $N$ behavior of the integral (8.2) in a standard fashion (it will be convenient to assume that $N$ is even). First of all, for large $N$, there should be no difference between $S O(N)$ and $O(N)$. An $S O(N)$ matrix can always be put in the blockdiagonal form with $2 \times 2$ blocks corresponding to a rotation by an angle $\alpha^{i}$ in the two-dimensional plane. Including the $S O(N)$ measure [54], the partition function (8.2) can be rewritten as

$$
\begin{aligned}
\mathcal{Z}= & \int \prod_{r=1}^{3} d \alpha_{r}^{i} \prod_{i<j}^{N / 2} \sin ^{2} \frac{\alpha_{r}^{i}-\alpha_{r}^{j}}{2} \sin ^{2} \frac{\alpha_{r}^{i}+\alpha_{r}^{j}}{2} \\
& \times \exp \left(8 \sum_{m=1}^{\infty} \frac{z\left(x^{m}\right)}{m} \prod_{r=1}^{3} \sum_{i=1}^{N / 2} \cos \left(m \alpha_{r}^{i}\right)\right) \\
= & \int[d \alpha] e^{-S_{\text {eff }}} .
\end{aligned}
$$

Index $r$ labels different $S O(N)_{r}$ groups, and $i, j=$ $1, \ldots, N / 2$ go over rotation angles. Also, we have introduced a single-letter partition function $z(x)$ to work in more generality. The above equation is valid for scalars, while for fermions, we need to include the factor $(-1)^{m+1}$ in front of $z\left(x^{m}\right)$. However, we will see in a moment that for the Hagedorn transition only the $m=1$ term is relevant. Therefore, our main results will be applicable for both cases.

The effective action $S_{\text {eff }}$ reads

$$
\begin{aligned}
S_{\mathrm{eff}}= & -\frac{1}{2} \sum_{r=1}^{3} \sum_{i \neq j}^{N / 2}\left(\log \sin ^{2} \frac{\alpha_{r}^{i}-\alpha_{r}^{j}}{2}+\log \sin ^{2} \frac{\alpha_{r}^{i}+\alpha_{r}^{j}}{2}\right) \\
& -8 \sum_{m=1}^{\infty} \frac{z\left(x^{m}\right)}{m} \prod_{r=1}^{3} \sum_{i=1}^{N / 2} \cos \left(m \alpha_{r}^{i}\right) .
\end{aligned}
$$

There are three saddle-point equations. One of them is

$$
\begin{aligned}
& \sum_{j=1}^{N / 2}\left(\cot \frac{\alpha_{1}^{i}-\alpha_{1}^{j}}{2}+\cot \frac{\alpha_{1}^{i}+\alpha_{1}^{j}}{2}\right) \\
& \quad-8 \sum_{m=1}^{\infty} z\left(x^{m}\right) \sin \left(m \alpha_{1}^{i}\right) \sum_{j_{2}, j_{3}} \cos \left(m \alpha_{2}^{j_{2}}\right) \cos \left(m \alpha_{3}^{j_{3}}\right)=0 .
\end{aligned}
$$

The other two can be obtained by cyclic permutations of $\alpha_{1}^{i}, \alpha_{2}^{i}, \alpha_{3}^{i}$. Introducing density functions, 


$$
\rho_{r}(\alpha)=\frac{2}{N} \sum_{i=1}^{N / 2} \delta\left(\alpha-\alpha_{r}^{i}\right)
$$

The saddle-point equation can be rewritten as

$$
\begin{gathered}
\int_{-\pi}^{\pi} d \alpha_{1}^{\prime} \rho_{1}\left(\alpha_{1}^{\prime}\right)\left(\cot \frac{\alpha_{1}-\alpha_{1}^{\prime}}{2}+\cot \frac{\alpha_{1}+\alpha_{1}^{\prime}}{2}\right) \\
-4 N \sum_{m=1}^{\infty} z\left(x^{m}\right) \sin \left(m \alpha_{1}\right) \rho_{2}^{m} \rho_{3}^{m}=0,
\end{gathered}
$$

where

$$
\rho_{r}^{m}=\int_{-\pi}^{\pi} d \alpha \rho_{r}(\alpha) \cos (m \alpha)
$$

It is natural to assume that, because of the cyclic symmetry, $\rho_{1}=\rho_{2}=\rho_{3}=\rho(\alpha)$. Moreover, we will assume that $\rho$ is an even function: $\rho(\alpha)=\rho(-\alpha)$. With these assumptions, the saddle-point equation reads as

$2 \int_{-\pi}^{\pi} d \alpha^{\prime} \rho\left(\alpha^{\prime}\right) \cot \frac{\alpha-\alpha^{\prime}}{2}-4 N \sum_{m=1}^{\infty} z\left(x^{m}\right) \sin (m \alpha)\left(\rho^{m}\right)^{2}=0$.

This is exactly the saddle-point equation studied in Ref. [40], with their $6 N$ replaced by our $2 N$. They have found that there is Hagedorn transition; for low temperatures when $N z(x)<27 / 16$, the partition function is dominated by the uniform saddle

$$
\rho(\alpha)=\frac{1}{2 \pi}, \quad \alpha \in[-\pi, \pi] .
$$

And so, all $\rho^{m}$ are zero for $m>0$. For higher temperatures, the density $\rho$ is not a constant and takes nonzero values only within a smaller interval $\left[-\alpha_{0}, \alpha_{0}\right]$. Moreover, the transition point itself can be found by assuming that only $\rho^{1}$ becomes nonzero. Therefore, the transition takes place at $N z(x)=$ $27 / 16$ for both bosons and fermions as we have advertised above. More details can be found in Refs. $[39,40]$.

For example, we can study the fermions in $d=1+2 \epsilon$. According to Eq. (7.12), in the UV, the transition happens at

$$
z_{F, 1+2 \epsilon}=x^{\epsilon}=\exp (-\beta \epsilon)=\frac{27}{16 N} .
$$

In the IR, the fermions have dimension $1 / 4$ for $d=1$. Assuming that most $2 k$-fermion operators have dimension $k / 2$, we conclude that the transition takes place at

$$
z_{F, \mathrm{IR}}=x^{1 / 4}=\exp (-\beta / 4)=\frac{27}{16 N} .
$$

\section{ACKNOWLEDGMENTS}

We are very grateful to $\mathrm{E}$. Witten for important input into many aspects of this paper. We are also grateful to S. Minwalla for important discussions and for sharing a draft of the paper [48] prior to publication. We also thank I. Danilenko, A. Jevicki, C. Krishnan, J. Maldacena, C. Peng, F. Popov, D. Roberts, S. Shenker, and D. Stanford for useful discussions. The work of I. R. K. and G. T. was supported in part by the U.S. NSF under Grant No. PHY1620059. G. T. acknowledges the support of a MyhrvoldHavranek Innovative Thinking Fellowship.
[1] R. Gurau, Colored group field theory, Commun. Math. Phys. 304, 69 (2011).

[2] R. Gurau and J.P. Ryan, Colored Tensor Models-A review, SIGMA 8, 020 (2012).

[3] R. Gurau and V. Rivasseau, The 1/N expansion of colored tensor models in arbitrary dimension, Europhys. Lett. 95, 50004 (2011).

[4] R. Gurau, The complete $1 / N$ expansion of colored tensor models in arbitrary dimension, Ann. Inst. Henri Poincaré 13, 399 (2012).

[5] V. Bonzom, R. Gurau, A. Riello, and V. Rivasseau, Critical behavior of colored tensor models in the large $\mathrm{N}$ limit, Nucl. Phys. B853, 174 (2011).

[6] A. Tanasa, Multi-orientable group field theory, J. Phys. A 45, 165401 (2012).
[7] V. Bonzom, R. Gurau, and V. Rivasseau, Random tensor models in the large $\mathrm{N}$ limit: Uncoloring the colored tensor models, Phys. Rev. D 85, 084037 (2012).

[8] S. Dartois, V. Rivasseau, and A. Tanasa, The $1 / N$ expansion of multi-orientable random tensor models, Ann. Inst. Henri Poincaré 15, 965 (2014).

[9] A. Tanasa, The multi-orientable random tensor model, a review, SIGMA 12, 056 (2016).

[10] S. Carrozza and A. Tanasa, $O(N)$ random tensor models, Lett. Math. Phys. 106, 1531 (2016).

[11] R. Gurau, The complete $1 / N$ expansion of a SYK-like tensor model, Nucl. Phys. B916, 386 (2017).

[12] I. R. Klebanov and G. Tarnopolsky, On large $N$ limit of symmetric traceless tensor models, J. High Energy Phys. 10 (2017) 037. 
[13] R. Gurau, The $1 / N$ expansion of tensor models with two symmetric tensors, arXiv:1706.05328.

[14] J. Ambjorn, B. Durhuus, and T. Jonsson, Three-dimensional simplicial quantum gravity and generalized matrix models, Mod. Phys. Lett. A 06, 1133 (1991).

[15] N. Sasakura, Tensor model for gravity and orientability of manifold, Mod. Phys. Lett. A 06, 2613 (1991).

[16] M. Gross, Tensor models and simplicial quantum gravity in > 2-D, Nucl. Phys. B, Proc. Suppl. 25A, 144 (1992).

[17] E. Witten, An SYK-Like Model Without Disorder, arXiv:1610.09758.

[18] I. R. Klebanov and G. Tarnopolsky, Uncolored random tensors, melon diagrams, and the Sachdev-Ye-Kitaev models, Phys. Rev. D 95, 046004 (2017).

[19] S. Sachdev and J. Ye, Gapless Spin Fluid Ground State in a Random, Quantum Heisenberg Magnet, Phys. Rev. Lett. 70, 3339 (1993).

[20] O. Parcollet and A. Georges, Non-Fermi-liquid regime of a doped Mott insulator, Phys. Rev. B 59, 5341 (1999).

[21] A. Georges, O. Parcollet, and S. Sachdev, Mean Field Theory of a Quantum Heisenberg Spin Glass, Phys. Rev. Lett. 85, 840 (2000).

[22] A. Kitaev, in Talks at KITP, LOCATION, 2015 (unpublished), http://online.kitp.ucsb.edu/online/entangled15/ kitaev/; and in Talks at KITP, LOCATION, 2015 (unpublished), http://online.kitp.ucsb.edu/online/entangled15/ kitaev2/.

[23] D. J. Gross and V. Rosenhaus, A generalization of SachdevYe-Kitaev, J. High Energy Phys. 02 (2017) 093.

[24] F. Ferrari, The Large D Limit of Planar Diagrams, arXiv:1701.01171.

[25] J. Polchinski and V. Rosenhaus, The spectrum in the Sachdev-Ye-Kitaev model, J. High Energy Phys. 04 (2016) 001.

[26] J. Maldacena and D. Stanford, Comments on the SachdevYe-Kitaev model, Phys. Rev. D 94, 106002 (2016).

[27] A. Jevicki, K. Suzuki, and J. Yoon, Bi-local holography in the SYK model, J. High Energy Phys. 07 (2016) 007.

[28] J. Maldacena, D. Stanford, and Z. Yang, Conformal symmetry and its breaking in two dimensional nearly antide-Sitter space, Prog. Theor. Exp. Phys. 2016, 12C104 (2016).

[29] J. Engelsoy, T. G. Mertens, and H. Verlinde, An investigation of $\mathrm{AdS}_{2}$ backreaction and holography, J. High Energy Phys. 07 (2016) 139.

[30] K. Jensen, Chaos in $\mathrm{AdS}_{2}$ Holography, Phys. Rev. Lett. 117, 111601 (2016).

[31] W. Fu, D. Gaiotto, J. Maldacena, and S. Sachdev, Supersymmetric SYK models, Phys. Rev. D 95, 026009 (2017).

[32] R. A. Davison, W. Fu, A. Georges, Y. Gu, K. Jensen, and S. Sachdev, Thermoelectric transport in disordered metals without quasiparticles: The Sachdev-Ye-Kitaev models and holography, Phys. Rev. B 95, 155131 (2017).

[33] J. Murugan, D. Stanford, and E. Witten, More on supersymmetric and $2 \mathrm{~d}$ analogs of the SYK model, J. High Energy Phys. 08 (2017) 146.
[34] C. Peng, M. Spradlin, and A. Volovich, Correlators in the $\mathcal{N}=2$ supersymmetric SYK model, J. High Energy Phys. 10 (2017) 202.

[35] K. Bulycheva, A note on the SYK model with complex fermions, J. High Energy Phys. 12 (2017) 069.

[36] J. Yoon, SYK models and SYK-like tensor models with global symmetry, J. High Energy Phys. 10 (2017) 183.

[37] B. Sundborg, The Hagedorn transition, deconfinement and $N=4$ SYM theory, Nucl. Phys. B573, 349 (2000).

[38] A. M. Polyakov, Gauge fields and space-time, Int. J. Mod. Phys. A 17, 119 (2002).

[39] O. Aharony, J. Marsano, S. Minwalla, K. Papadodimas, and M. Van Raamsdonk, The Hagedorn-deconfinement phase transition in weakly coupled large $\mathrm{N}$ gauge theories, Adv. Theor. Math. Phys. 8, 603 (2004).

[40] M. Beccaria and A. A. Tseytlin, Partition function of free conformal fields in 3-plet representation, J. High Energy Phys. 05 (2017) 053.

[41] D. Boulatov and V. Kazakov, One-dimensional string theory with vortices as the upside down matrix oscillator, Int. J. Mod. Phys. A 08, 809 (1993).

[42] J. B. Geloun and S. Ramgoolam, , Counting Tensor Model Observables and Branched Covers of the 2-Sphere, arXiv:1307.6490.

[43] H. Itoyama, A. Mironov, and A. Morozov, Ward identities and combinatorics of rainbow tensor models, J. High Energy Phys. 06 (2017) 115.

[44] A. Mironov and A. Morozov, Correlators in tensor models from character calculus, Phys. Lett. B 774, 210 (2017).

[45] P. Diaz and S.-J. Rey, Orthogonal Bases of Invariants in Tensor Models, arXiv:1706.02667.

[46] R. de Mello Koch, D. Gossman, and L. Tribelhorn, Gauge invariants, correlators and holography in bosonic and fermionic tensor models, J. High Energy Phys. 09 (2017) 011.

[47] V. Bonzom, L. Lionni, and A. Tanasa, Diagrammatics of a colored SYK model and of an SYK-like tensor model, leading and next-to-leading orders, J. Math. Phys. (N.Y.) 58, 052301 (2017).

[48] S. Choudhury, A. Dey, I. Halder, L. Janagal, S. Minwalla, and R. Poojary, Notes on Melonic $O(N)^{q-1}$ Tensor Models, arXiv:1707.09352.

[49] I. Klebanov, D. Roberts, D. Stanford, and G. Tarnopolsky (unpublished).

[50] C. Krishnan, K. V. P. Kumar, and S. Sanyal, Random matrices and holographic tensor models, J. High Energy Phys. 06 (2017) 036.

[51] C. Krishnan and K. V. P. Kumar, Towards a finite- $N$ hologram, J. High Energy Phys. 10 (2017) 099.

[52] E. Witten, in Symposium on Anomalies, Geometry, Topology, Argonne, IL, 1985 (unpublished).

[53] S. Elitzur, Y. Frishman, E. Rabinovici, and A. Schwimmer, Origins of global anomalies in quantum mechanics, Nucl. Phys. B273, 93 (1986).

[54] W. Krauth and M. Staudacher, Yang-Mills integrals for orthogonal, symplectic and exceptional groups, Nucl. Phys. B584, 641 (2000). 